\title{
Scenarios for gluino coannihilation
}

\author{
John Ellis, ${ }^{a, b}$ Jason L. Evans, ${ }^{c, d}$ Feng Luo $^{b}$ and Keith A. Olive $e^{c, d}$ \\ ${ }^{a}$ Theoretical Particle Physics and Cosmology Group, Department of Physics, \\ King's College London, London, WC2R 2LS United Kingdom \\ ' Theory Division, CERN, \\ Geneva 23, CH-1211 Switzerland \\ ${ }^{c}$ School of Physics and Astronomy, University of Minnesota, \\ Minneapolis, MN, 55455 U.S.A. \\ ${ }^{d}$ William I. Fine Theoretical Physics Institute, School of Physics and Astronomy, \\ University of Minnesota, Minneapolis, MN, 55455 U.S.A. \\ E-mail: john.ellis@cern.ch, jlevans@umn.edu, f.luo@cern.ch, \\ olive@physics.umn.edu
}

ABSTRACT: We study supersymmetric scenarios in which the gluino is the next-to-lightest supersymmetric particle (NLSP), with a mass sufficiently close to that of the lightest supersymmetric particle (LSP) that gluino coannihilation becomes important. One of these scenarios is the MSSM with soft supersymmetry-breaking squark and slepton masses that are universal at an input GUT renormalization scale, but with non-universal gaugino masses. The other scenario is an extension of the MSSM to include vector-like supermultiplets. In both scenarios, we identify the regions of parameter space where gluino coannihilation is important, and discuss their relations to other regions of parameter space where other mechanisms bring the dark matter density into the range allowed by cosmology. In the case of the non-universal MSSM scenario, we find that the allowed range of parameter space is constrained by the requirement of electroweak symmetry breaking, the avoidance of a charged LSP and the measured mass of the Higgs boson, in particular, as well as the appearance of other dark matter (co)annihilation processes. Nevertheless, LSP masses $m_{\chi} \lesssim 8 \mathrm{TeV}$ with the correct dark matter density are quite possible. In the case of pure gravity mediation with additional vector-like supermultiplets, changes to the anomalymediated gluino mass and the threshold effects associated with these states can make the gluino almost degenerate with the LSP, and we find a similar upper bound.

KEYWORds: Supersymmetry Phenomenology

ARXIV EPRINT: 1510.03498 


\section{Contents}

1 Introduction 1

2 Calculations of gluino coannihilation 3

3 The non-universal MSSM scenario $\quad 6$

4 Pure gravity mediation with vector multiplets $\quad 15$

$\begin{array}{lll}5 & \text { Summary and conclusions } & 19\end{array}$

\section{Introduction}

The absence of supersymmetry so far, at LHC Run I $[1-3]^{1,2}$ and elsewhere, raises the question where, if anywhere, is it hiding. There are scenarios for which, at least, some supersymmetric particles were produced in LHC Run I, but have been overlooked. Examples include models where $R$-parity is violated $[4,5]$, or the spectra are compressed [6-9]. Alternatively, sparticles might be too heavy to have been detected at LHC Run I, but might be within range of future LHC runs [10]. It is also possible that supersymmetric particles may lie beyond the reach of the LHC altogether, and require a future higher-energy $p p$ collider for their detection.

If one assumes that $R$-parity is conserved, the lightest supersymmetric particle (LSP) must be stable, and hence makes at least a contribution to the cosmological cold dark matter density $[11,12]$. The total density of cold dark matter is very tightly constrained by measurements of the cosmic microwave background radiation [13]. It is clear, therefore, that the parameters of generic models are constrained in very specific ways in order to realize the correct dark matter density [14-31]. Moreover, this parameter space with the correct density is likely to be found in a region of parameter space where the density varies rapidly with the parameters. In these cases, regions where the LSP contributes only a fraction of the cold dark matter density will have parameters similar to those regions yielding the correct total density.

This sensitivity of the dark matter density to parameters are particularly relevant for models with compressed and/or very heavy spectra that have survived LHC searches. Examples of specific choices of heavy spectra that yield the correct cosmological dark matter density include scenarios in which the LSP, $\chi$, would have annihilated with itself

\footnotetext{
${ }^{1}$ Full ATLAS Run 1 results can be found at https://twiki.cern.ch/twiki/bin/view/AtlasPublic/SupersymmetryPublicResults.

${ }^{2}$ Full CMS Run 1 results can be found at https://twiki.cern.ch/twiki/bin/view/CMSPublic/PhysicsResultsSUS.
} 
through a direct-channel boson such as the heavier neutral Higgs bosons $A$ and $H[32-$ 36]. Alternatively, there might be one or more heavier supersymmetric particles that are nearly degenerate with the LSP, $\chi$, and would have coannihilated with it in the early Universe [37]. There are several examples of possible coannihilating sparticles, including the lighter stau, or possibly some other slepton [38-45], the lighter stop squark [46-56], the lighter chargino [55, 57-60] and the gluino [56, 61-72].

In most cases, coannihilation with a sparticle having stronger interactions extends the allowed mass range of the LSP. The possibility of gluino coannihilation is therefore particularly interesting since it interacts strongly suggesting it can accommodate a heavier LSP than is possible from coannihilation with a stau or slepton. In fact, it has been shown that a dark matter density realized by an LSP coannihilating with the gluino could lie well beyond the reach of the LHC, with a mass as heavy as $m_{\chi} \lesssim 8 \mathrm{TeV}[72]$.

The possibility of gluino coannihilation does not arise in the minimal supersymmetric extension of the Standard Model (MSSM) with the soft supersymmetry-breaking parameters constrained to be universal at the input GUT scale (the CMSSM) [14, 32-36, 73-92], nor in related models with non-universal Higgs masses [83, 84, 93-106]. However, as we discuss in this paper, gluino coannihilation can become important in variants of the MSSM with non-universal gaugino masses, and in variations of pure gravity mediation (PGM) with non-minimal matter content such as additional vector-like supermultiplets [67-69].

We use the SSARD (SuperSymmetry And Relic Density) code ${ }^{3}$ to calculate the particle spectrum and relic density. SSARD first calculates the supersymmetric particle spectrum for a given set of boundary conditions defined by the model - the modified CMSSM or PGM. Coupled renormalization-group equations (RGEs) are then run back and forth between the weak scale and the GUT scale, which is defined by the renormalization scale where the two electroweak gauge couplings are equal. The gauge and Yukawa couplings are run at two loops, whereas the soft supersymmetry-breaking masses are run at one loop. The strong gauge coupling is fixed at the weak scale. Once convergence of the RGEs is obtained, the sfermion mass matrices are evaluated to obtain physical masses. SSARD determines $\mu$ and $B \mu$ at the weak scale by minimizing the Higgs tadpole equations. We calculate the Higgs mass using the procedure outlined in [107, 108]. ${ }^{4}$ The neutralino and chargino mass matrices are then diagonalized with one-loop corrections applied. With the sparticle spectrum determined, the cross sections for annihilation and co-annihilation are computed and input into a routine that integrates numerically the Boltzmann equation to determine the cosmological relic density. Finally, branching fractions for rare decays are computed, along with the value of $g_{\mu}-2$ and the neutralino-nucleon scattering cross section.

\footnotetext{
${ }^{3}$ Information about this code is available from K.A. Olive: it contains important contributions from J. Evans, T. Falk, A. Ferstl, G. Ganis, F. Luo, A. Mustafayev, J. McDonald, K.A. Olive, P. Sandick, Y. Santoso, V. Spanos, and M. Srednicki.

${ }^{4}$ This differs from most implementations of SSARD, which often uses FeynHiggs [109-114] to calculate the Higgs mass. However, FeynHiggs loses stability at mass scales significantly above $10 \mathrm{TeV}$ let alone the order 100-1000 TeV mass scales considered here. In the implementation used here, the Higgs mass is computed from its effective quartic coupling as discussed in [107, 108]. For more information on how the Higgs mass calculation is used in SSARD see [115].
} 
The layout of this paper is as follows. In section 2 we set out the coupled set of Boltzmann equations that we use to calculate the relic LSP density, discussing the circumstances under which the analysis can be reduced to a single Boltzmann equation for a particular combination of sparticle abundances [72]. Then, in section 3 we discuss various scenarios with non-universal gaugino masses in which gluino coannihilation can become important, delineating the corresponding strips in parameter space and comparing their extents with the results of [72]. We find that this scenario is constrained by the requirement of consistent electroweak symmetry breaking (EWSB), by the measurement of $m_{H}$, and by avoidance of a stop or chargino LSP. We give examples showing that the correct dark matter density is possible with LSP masses as large as $8 \mathrm{TeV}$. Section 4 contains a similar analysis of PGM models with vector-like supermultiplets, focusing on an example with a single extra pair of $\mathbf{1 0}$ and $\overline{\mathbf{1 0}}$ representations of SU(5). Because the anomaly-mediated contribution to the gluino mass is zero in this case, threshold effects due to these additional states generate almost the entire gluino mass. This suppresses the gluino mass relative to those of the other gauginos, leading to near-degeneracy between the gluino and the LSP. Neutralino dark matter candidates with similarly large values of $m_{\chi}$ are again possible. Finally, section 5 summarizes our conclusions and discusses the prospects for discovering supersymmetry in these gluino coannihilation scenarios.

\section{Calculations of gluino coannihilation}

In this section, we present general formulae for calculating the dark matter thermal relic density, and then specialize it to the case of the gluino coannihilation scenarios we consider in this paper, taking into account the effects of gluino-gluino bound states.

We consider $N R$-odd species in the thermal bath in the early Universe. We assume that the rates for interconverting the LSP (which is labeled as the first species, with mass $\left.m_{1}\right)$ and the first $l$ species $(1 \leq l \leq N)$ are sufficiently large, compared to the Hubble expansion rate, that to a very good approximation the ratios of densities are equal to the equilibrium ratios: $n_{i} / n_{1}=n_{i}^{\text {eq }} / n_{1}^{\text {eq }}$ for $(i=1, \ldots, l)$.

For any of the $N$ species, the evolution of its number density is governed by the Boltzmann equation

$$
\frac{d n_{i}}{d t}+3 H(T) n_{i}=-\sum_{j=1}^{N}\langle\sigma v\rangle_{i j \rightarrow \mathrm{SM}}\left(n_{i} n_{j}-n_{i}^{\mathrm{eq}} n_{j}^{\mathrm{eq}}\right)-\sum_{\substack{j=1 \\ j n_{\mathrm{eq}} i}}^{N}\langle\Gamma\rangle_{i \rightarrow j}\left(n_{i}-n_{i}^{\mathrm{eq}} \frac{n_{j}}{n_{j}^{\mathrm{eq}}}\right),
$$

where $\langle\sigma v\rangle_{i j \rightarrow \mathrm{SM}}$ is the product of the thermally-averaged relative velocity with the total cross section for the channels of $i$ and $j$ (co)annihilating into Standard Model particles, and $\langle\Gamma\rangle_{i \rightarrow j}$ is the sum of all the thermally-averaged decay and conversion rates for decay and conversion processes for which there is one particle $i$ in the initial state and one particle $j$ in the final state, with all other particles involved in these processes being Standard Model particles. The relations between the thermally-averaged forward and backward reactions are used in (2.1), and we assume for all the Standard Model particles involved 
that $n_{\mathrm{SM}}=n_{\mathrm{SM}}^{\mathrm{eq}}$, so that, for example,

$$
\langle\Gamma\rangle_{i \rightarrow j} n_{i}^{\mathrm{eq}}=\langle\Gamma\rangle_{j \rightarrow i} n_{j}^{\mathrm{eq}} .
$$

Written in terms of the yields, $Y_{i} \equiv n_{i} / s,(2.1)$ becomes

$$
\frac{d Y_{i}}{d x}=-\frac{x s}{H\left(m_{1}\right)}\left(1+\frac{T}{3 g_{* s}} \frac{d g_{* s}}{d T}\right)\left[\sum_{j=1}^{N}\langle\sigma v\rangle_{i j \rightarrow \mathrm{SM}}\left(Y_{i} Y_{j}-Y_{i}^{\mathrm{eq}} Y_{j}^{\mathrm{eq}}\right)+\sum_{\substack{j=1 \\ j n_{\mathrm{eq}} i}}^{N} \frac{\langle\Gamma\rangle_{i \rightarrow j}}{s}\left(Y_{i}-Y_{i}^{\mathrm{eq}} \frac{Y_{j}}{Y_{j}^{\mathrm{eq}}}\right)\right],
$$

where

$$
x \equiv \frac{m_{1}}{T}, \quad s=\frac{2 \pi^{2}}{45} g_{* s} T^{3}, \quad H\left(m_{1}\right) \equiv H(T) x^{2}=\left(\frac{4 \pi^{3} G_{N} g_{*}}{45}\right)^{\frac{1}{2}} m_{1}^{2},
$$

and $g_{* s}$ and $g_{*}$ are the total numbers of effectively massless degrees of freedom associated with the entropy density and the energy density, respectively.

Defining $\tilde{Y} \equiv \sum_{i=1}^{l} Y_{i}, \Delta_{i} \equiv\left(m_{i}-m_{1}\right) / m_{1}$ and $\tilde{g}_{\text {eff }} \equiv \sum_{i=1}^{l} g_{i}\left(1+\Delta_{i}\right)^{3 / 2} e^{-\Delta_{i} x}$, we have

$$
\frac{Y_{i}^{\mathrm{eq}}}{\tilde{Y}^{\mathrm{eq}}}=\frac{g_{i}\left(\frac{m_{i} T}{2 \pi}\right)^{3 / 2} e^{-m_{i} / T}}{\sum_{j=1}^{l} g_{j}\left(\frac{m_{j} T}{2 \pi}\right)^{3 / 2} e^{-m_{j} / T}}=\frac{g_{i}\left(1+\Delta_{i}\right)^{3 / 2} e^{-\Delta_{i} x}}{\tilde{g}_{\mathrm{eff}}} .
$$

Using $Y_{i} / \tilde{Y}=Y_{i}^{\mathrm{eq}} / \tilde{Y}^{\mathrm{eq}}$ for $i=1, \ldots, l$ and summing over $(2.3)$ for the first $l$ species, we find

$$
\begin{aligned}
\frac{d \tilde{Y}}{d x}= & -\frac{x s}{H\left(m_{1}\right)}\left(1+\frac{T}{3 g_{* s}} \frac{d g_{* s}}{d T}\right)\left\{\left\langle\tilde{\sigma}_{\mathrm{eff}} v\right\rangle\left(\tilde{Y}^{2}-\tilde{Y}_{\mathrm{eq}}^{2}\right)\right. \\
& \left.+\sum_{k=l+1}^{N}\left[\left\langle\sigma_{k \mathrm{eff}} v\right\rangle\left(\tilde{Y} Y_{k}-\tilde{Y}^{\mathrm{eq}} Y_{k}^{\mathrm{eq}}\right)+\frac{\left\langle\Gamma_{k}\right\rangle_{\mathrm{eff}}}{s}\left(\tilde{Y}-\tilde{Y}^{\mathrm{eq}} \frac{Y_{k}}{Y_{k}^{\mathrm{eq}}}\right)\right]\right\},
\end{aligned}
$$

while for each of the species $k(l<k \leq N)$, we get

$$
\begin{aligned}
\frac{d Y_{k}}{d x}= & -\frac{x s}{H\left(m_{1}\right)}\left(1+\frac{T}{3 g_{* s}} \frac{d g_{* s}}{d T}\right)\left\{\left\langle\sigma_{k \mathrm{eff}} v\right\rangle\left(\tilde{Y} Y_{k}-\tilde{Y}^{\mathrm{eq}} Y_{k}^{\mathrm{eq}}\right)\right. \\
& +\sum_{j=l+1}^{N}\langle\sigma v\rangle_{k j \rightarrow \mathrm{SM}}\left(Y_{k} Y_{j}-Y_{k}^{\mathrm{eq}} Y_{j}^{\mathrm{eq}}\right)-\frac{\left\langle\Gamma_{k}\right\rangle_{\mathrm{eff}}}{s}\left(\tilde{Y}-\tilde{Y}^{\mathrm{eq}} \frac{Y_{k}}{Y_{k}^{\mathrm{eq}}}\right) \\
& \left.+\sum_{\substack{j=l+1 \\
j n_{\mathrm{eq}} k}}^{N} \frac{\langle\Gamma\rangle_{k \rightarrow j}}{s}\left(Y_{k}-Y_{k}^{\mathrm{eq}} \frac{Y_{j}}{Y_{j}^{\mathrm{eq}}}\right)\right\},
\end{aligned}
$$


where

$$
\begin{aligned}
\left\langle\sigma_{k \mathrm{eff}} v\right\rangle & \equiv \sum_{i=1}^{l}\langle\sigma v\rangle_{i k \rightarrow \mathrm{SM}} \frac{Y_{i}^{\mathrm{eq}}}{\tilde{Y}^{\mathrm{eq}}}=\sum_{i=1}^{l}\langle\sigma v\rangle_{i k \rightarrow \mathrm{SM}} \frac{g_{i}\left(1+\Delta_{i}\right)^{3 / 2} e^{-\Delta_{i} x}}{\tilde{g}_{\mathrm{eff}}}, \\
\left\langle\Gamma_{k}\right\rangle_{\mathrm{eff}} & \equiv \sum_{i=1}^{l}\langle\Gamma\rangle_{i \rightarrow k} \frac{Y_{i}^{\mathrm{eq}}}{\tilde{Y}^{\mathrm{eq}}}=\sum_{i=1}^{l}\langle\Gamma\rangle_{i \rightarrow k} \frac{g_{i}\left(1+\Delta_{i}\right)^{3 / 2} e^{-\Delta_{i} x}}{\tilde{g}_{\mathrm{eff}}}, \\
\left\langle\tilde{\sigma}_{\mathrm{eff}} v\right\rangle & \equiv \sum_{i, j=1}^{l}\langle\sigma v\rangle_{i j \rightarrow \mathrm{SM}} \frac{Y_{i}^{\mathrm{eq}} Y_{j}^{\mathrm{eq}}}{\tilde{Y}_{\mathrm{eq}}^{2}}=\sum_{i, j=1}^{l}\langle\sigma v\rangle_{i j \rightarrow \mathrm{SM}} \frac{g_{i} g_{j}\left(1+\Delta_{i}\right)^{3 / 2}\left(1+\Delta_{j}\right)^{3 / 2} e^{-\left(\Delta_{i}+\Delta_{j}\right) x}}{\tilde{g}_{\mathrm{eff}}^{2}} .
\end{aligned}
$$

We note that in the case $N=l+1$, the final term in (2.7) does not appear. In the case $N=l,(2.6)$ does not have the two terms in the squared bracket, and reverts to the familiar form for coannihilations when all the $N$ species are sufficiently coupled to the LSP.

We now specialize the above general formulae to the gluino coannihilation scenarios we consider in this paper. First of all, following the discussion in [72], the effect of gluino-gluino bound states on the calculation of the dark matter relic density can be taken into account simply by modifying the Boltzmann equation by including the Sommerfeldenhanced thermal-averaged velocity-weighted gluino pair annihilation cross section [70], which includes gluino-pair annihilation to two gluons and to all the quark anti-quark pair channels:

$$
\langle\sigma v\rangle_{\tilde{g} \tilde{g} \rightarrow g g, q \bar{q}} \rightarrow\langle\sigma v\rangle_{\tilde{g} \tilde{g} \text { incl. } \tilde{R}} \equiv\langle\sigma v\rangle_{\tilde{g} \tilde{g} \rightarrow g g, q \bar{q}}+\langle\sigma v\rangle_{b s f} \frac{\langle\Gamma\rangle_{\tilde{R}}}{\langle\Gamma\rangle_{\tilde{R}}+\langle\Gamma\rangle_{\text {dis }}},
$$

where $\langle\sigma v\rangle_{b s f},\langle\Gamma\rangle_{\tilde{R}}$ and $\langle\Gamma\rangle_{\text {dis }}$ are the thermally-averaged formation cross section, decay rate and dissociation rate for the bound state $\tilde{R}$, respectively. The details of these quantities and the derivation of eq. (2.11) can be found in section 3,5 and appendix B of [72].

When the rate for interconverting the neutralino LSP and the gluino is sufficiently large, compared to the Hubble rate, so that to a good approximation the relation $Y_{\tilde{g}}(T) /$ $Y_{1}(T)=Y_{\tilde{g}}^{\mathrm{eq}}(T) / Y_{1}^{\mathrm{eq}}(T)$ holds at all temperatures during which the sum of $Y_{\tilde{g}}(T)$ and $Y_{1}(T)$ changes non-negligibly, we can use a single Boltzmann equation to solve for the dark matter relic abundance, including the gluino species in $\tilde{Y}$ and $\left\langle\tilde{\sigma}_{\text {eff }} v\right\rangle$ and using (2.6) without the two terms in the squared bracket. Otherwise, one should use a coupled set of Boltzmann equations, namely (2.6) and (2.7), to solve for the dark matter relic abundance. For the scenarios considered in this paper, any of the $R$-odd species apart from the gluino is either sufficiently coupled to the LSP by having a Standard Model particle in the propagator of a tree-level Feynman diagram describing its interconversion with the LSP, or is so heavy compared to the LSP that it is effectively not participating coannihilations. Therefore, when using a coupled set of Boltzmann equations, we have $N=l+1$ in (2.6) and (2.7), and the index $k$ is for the gluino.

We end this section by emphasizing that, in principle, the coupled set of Boltzmann equations can always be used to solve for the dark matter relic density, whether the rate for interconverting the gluino and the LSP is sufficiently large compared to the Hubble 
rate or not. However, for the former case, solving a single Boltzmann equation is usually easier than solving the coupled ones and requires less computing time.

\section{The non-universal MSSM scenario}

It was assumed in [72] that the squarks were all degenerate with a common mass $m_{\tilde{q}}$, and the effects of sparticles with only electroweak interactions were neglected. It was found in [72] that in the presence of gluino coannihilation, a Bino LSP, $\chi$, could be the dark matter of the universe if it weighed $\lesssim 8 \mathrm{TeV}$, the exact value depending on the ratio $m_{\tilde{q}} / m_{\chi}$, with smaller values of $m_{\chi}$ being found for $m_{\tilde{q}} / m_{\chi} \lesssim 5$ and $\gtrsim 100$. Here we make a more complete study in a variant of the MSSM with universal soft supersymmetry-breaking scalar masses $m_{0}$ and trilinear couplings $A_{0}$, allowing a restricted form of non-universality in the gaugino sector with $M_{1}=M_{2} \neq M_{3}$ at the input GUT scale. The results therefore depend on $M_{1} / M_{3}$ as well as the usual CMSSM parameters $m_{0}, A_{0}$ and $\tan \beta$ (the ratio of MSSM Higgs vev's). This is therefore a one-parameter extension of the CMSSM (with the new free parameter being $M_{3}$ ) as is the NUHM1 (with the soft Higgs masses $m_{1}=m_{2} \neq m_{0}$ ) [104-106]. We consider in this section various $\left(M_{1}, M_{3}\right)$ planes for various choices of the other parameters which illustrate the range of possibilities.

We first consider the example with $m_{0}=1000 \mathrm{TeV}, A_{0} / m_{0}=1.5$ and $\tan \beta=2.5$ shown in figure 1. In the left panel and in subsequent figures, the regions where the relic LSP density $\Omega_{\chi} h^{2}$ falls within the range allowed by Planck and other data are shown as dark blue strips, and the regions where the lightest neutralino is no longer the LSP are shaded brick-red. In this case, the gluino is the LSP in the shaded region. Because of the scale of the plot, it is difficult to discern the relic density strip, which lies very close to the boundary of this region. However, we note that it lies to the left of the red shaded region only when $M_{3}$ is between $\sim 400$ and $\sim 1200 \mathrm{GeV}$, as shown in the right panel of figure 1 by the left axis and blue curve, which shows the mass difference $\Delta M \equiv m_{\tilde{g}}-m_{\chi}$ between the gluino and the neutralino along the coannihilation strip as a function of the input gluino mass. Also shown in the right panel (as a red line) is the neutralino mass as a function of $M_{3}$.

The shapes of the blue and red curves in the right panel of figure 1 can be understood by comparing with figure 6 of [72], which shows the value of $m_{\chi}$ at the end-point of the gluino coannihilation strip when $\Delta M=0$ for a pure Bino LSP, as a function of the ratio of the assumed common squark mass, $m_{\tilde{q}}$, and $m_{\chi}$. We reproduce this plot here (figure 2 ) for the convenience of the reader. In this plot, the green band corresponds to the $3-\sigma$ range allowed by Planck and other data. The drop at small $m_{\tilde{q}} / m_{\chi}$ is due to the cancellations between the $s^{-}, t$ - and $u$-channel diagrams for gluino-pair annihilation into quark and antiquark, which results in a smaller annihilation cross section. The very rapid drop at large $m_{\tilde{q}} / m_{\chi}$ is due to the decoupling of the gluino and neutralino densities. In between there is a plateau with $m_{\chi} \sim 8 \mathrm{TeV}$ along the green band. As seen in figure 2 , the choice of $m_{0}$ in figure 1 corresponds to values of $m_{\tilde{q}} / m_{\chi}$ extending from beyond the plateau at small $M_{3}$ to values along the plateau at large $M_{3}$. The gluino coannihilation strip therefore has two end-points where $\Delta M \rightarrow 0$, corresponding to the limiting values $m_{\chi} \sim 6 \mathrm{TeV}$ and 

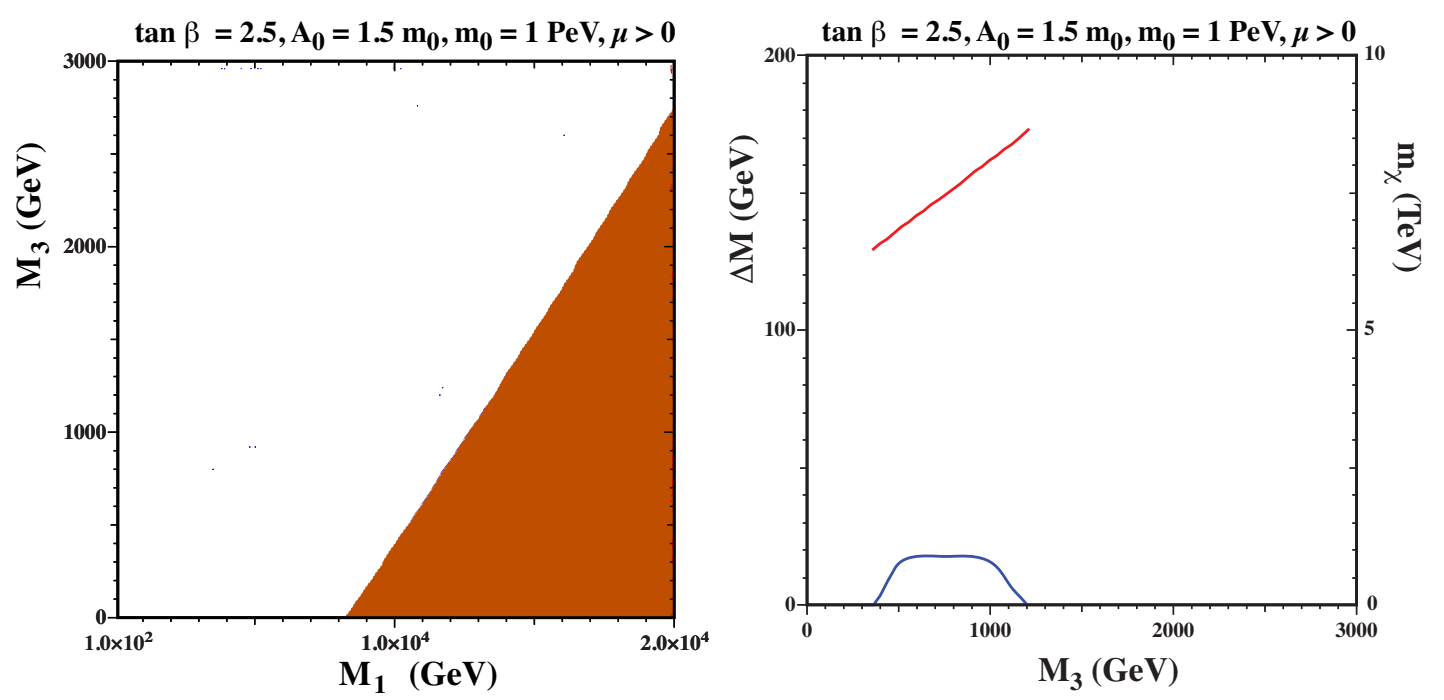

Figure 1. The $\left(M_{1}, M_{3}\right)$ plane (left) for $m_{0}=1000 \mathrm{TeV}, A_{0} / m_{0}=1.5$ and $\tan \beta=2.5$. The dark blue strip in the left panel shows where the relic LSP density $\Omega_{\chi} h^{2}$ falls within the $\pm 3-\sigma$ range allowed by Planck and other data, and the lightest neutralino is no longer the LSP in the regions shaded brick-red. The right panel shows the gluino-neutralino mass difference (left axis, blue line) and the neutralino mass (right axis, red line) as functions of $M_{3}$.

$m_{\chi} \sim 8 \mathrm{TeV}$ seen in the right panel of figure 1: for larger and smaller $M_{3}, \Delta M<0$ and the gluino is the LSP.

We list in the first column of table 1 some details of a sample parameter set from the model plane in figure 1 . The first few lines display the values of the input parameters $m_{0}, M_{1}, M_{3}, A_{0} / m_{0}$ and $\tan \beta$, followed by the Higgs mixing parameter $\mu$ derived from the electroweak vacuum conditions. The next few lines exhibit the masses of the gluino, neutralino LSP, lighter stop squark and Higgs boson, and the last two lines exhibit the spin-independent and -dependent LSP-proton scattering cross sections $\sigma_{\mathrm{SI}, \mathrm{SD}}$. All the mass parameters are given in $\mathrm{TeV}$ units, except for the Higgs mass, which is expressed in $\mathrm{GeV}$. The succeeding columns in table 1 show the corresponding numbers for parameter sets from the model planes shown in later figures. In the cases of the pure gravity mediation (PGM) models in the last two columns, the values of $c_{10}$ are quoted in parentheses $(\ldots)$.

We note that the Higgs mass is relatively insensitive to the choice of $M_{1}$ and $M_{3}$, and therefore varies very little across the plane with $\tan \beta, A_{0}$, and $m_{0}$ fixed. For the case shown in figure 1 , we calculate $m_{H} \approx 126.3 \mathrm{GeV}$, which is compatible with the experimental measurement, within the theoretical uncertainties. We do not show any other $\left(M_{1}, M_{3}\right)$ planes for $m_{0}=1000 \mathrm{TeV}$, since the possibilities are quite limited: there are no consistent solutions of the electroweak symmetry-breaking conditions for much smaller values of $A_{0} / m_{0} \lesssim 1$ and/or larger values of $\tan \beta$, and $m_{H}$ is too large for larger values of $\tan \beta$ and/or $A_{0} / m_{0}$ (though it increases quite slowly with $A_{0}$ ).

We consider next an example of a $\left(M_{1}, M_{3}\right)$ plane for $m_{0}=200 \mathrm{TeV}$, which corresponds to values of $m_{\tilde{q}} / m_{\chi}$ along the plateau in figure 2 . The left panel of figure 3 shows the $\left(M_{1}, M_{3}\right)$ plane for $\tan \beta=3$ and $A_{0} / m_{0}=1.5$. In this case there is a longer gluino 


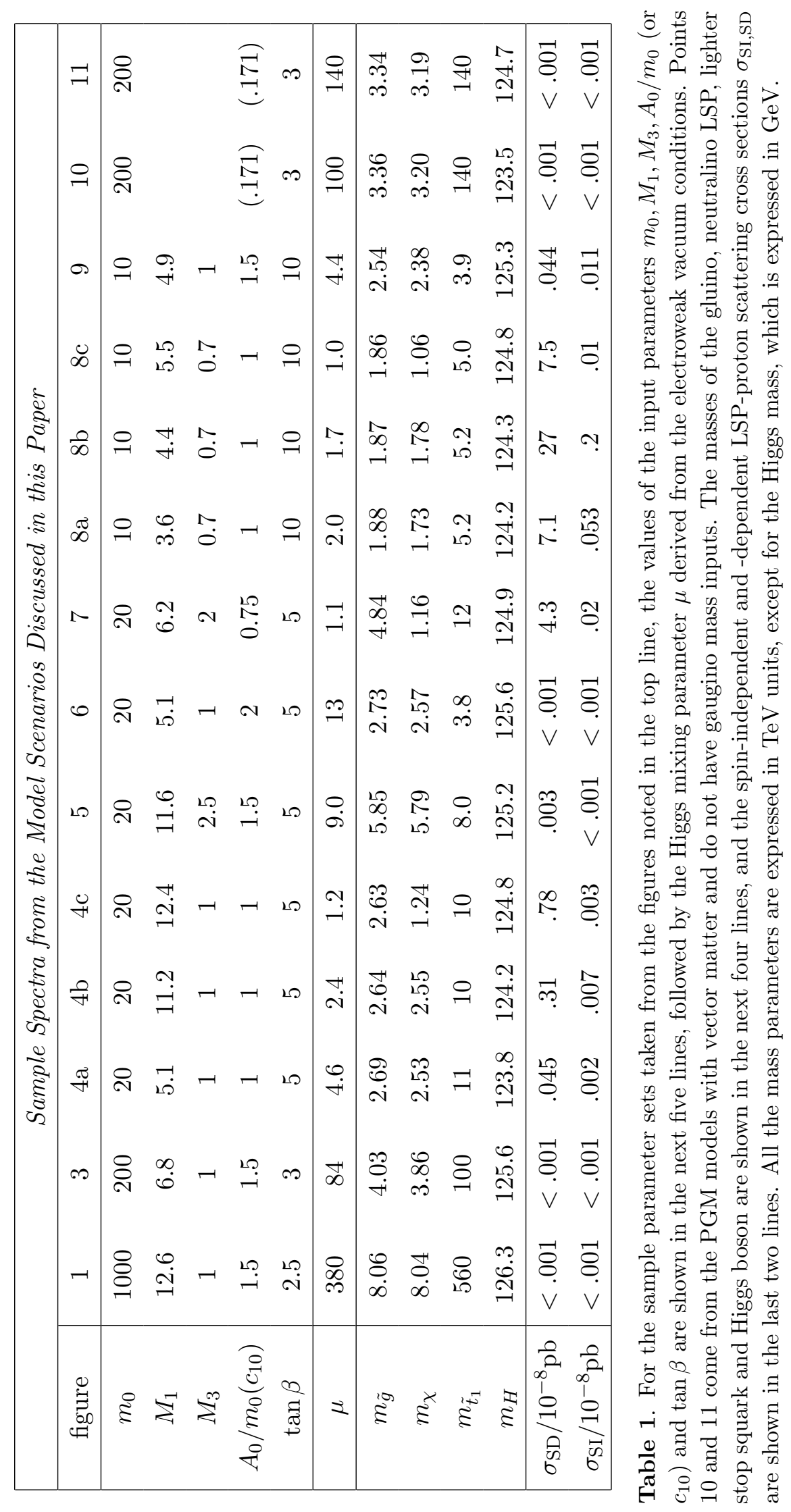




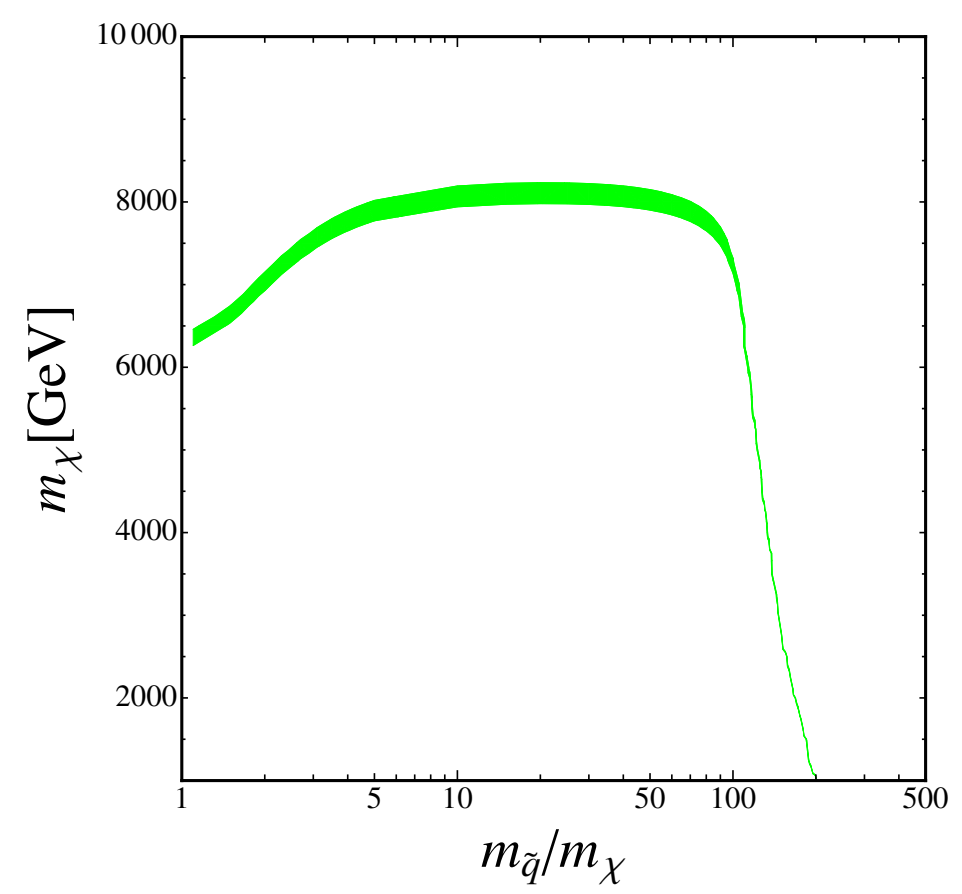

Figure 2. This figure is adapted from figure 6 of [72]. It shows the value of $m_{\chi}$ at the end-point of the gluino coannihilation strip when $\Delta M=0$ for a pure Bino LSP, as a function of $m_{\tilde{q}} / m_{\chi}$, where a common mass $m_{\tilde{q}}$ is assumed for all the squarks. The drop at small $m_{\tilde{q}} / m_{\chi}$ is due to the cancellations between the $s$-, $t$ - and $u$-channel diagrams for gluino-pair annihilation into quark and anti-quark, and that at large $m_{\tilde{q}} / m_{\chi}$ is due to the decoupling of the gluino and neutralino densities. The green band corresponds to the $3-\sigma$ range of the dark matter density: $\Omega_{\chi} h^{2}=0.1193 \pm 0.0042$.
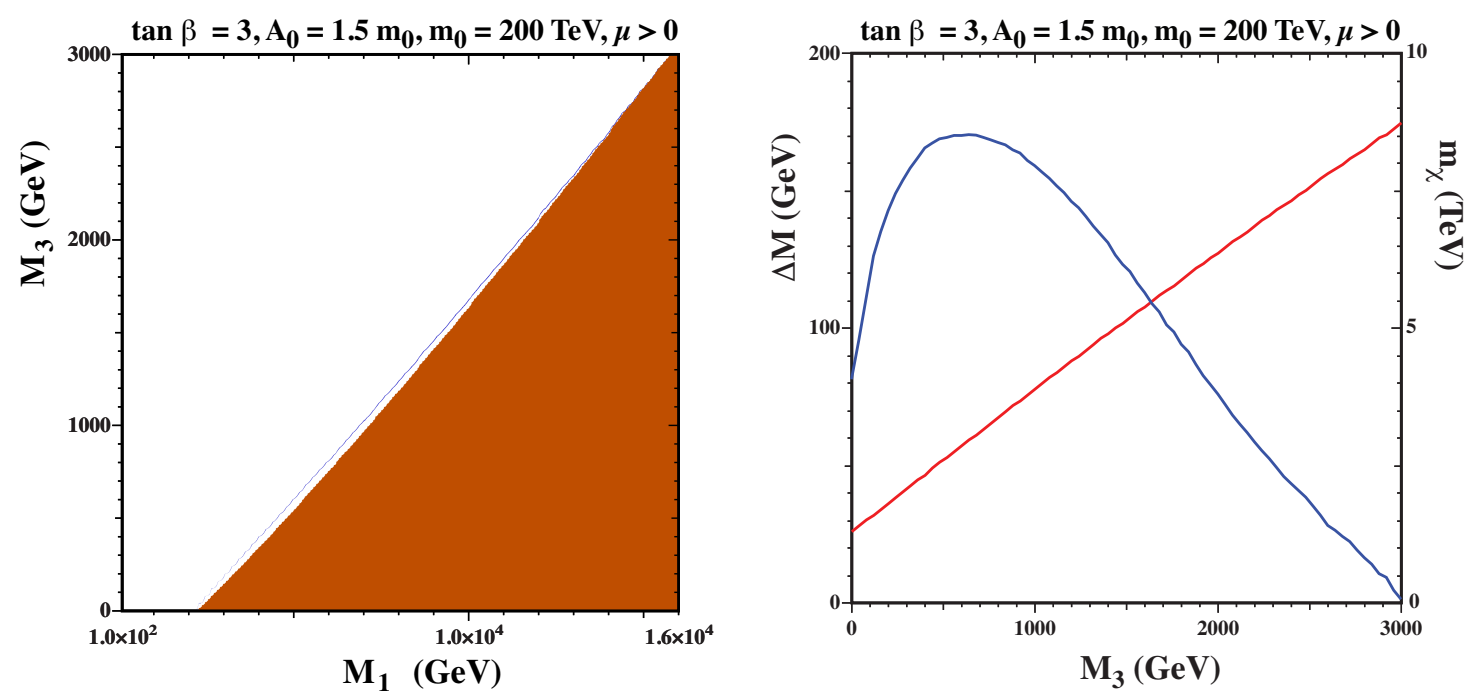

Figure 3. As for figure 1, but for $m_{0}=200 \mathrm{TeV}$ and $A_{0} / m_{0}=1.5$, with $\tan \beta=3$. 

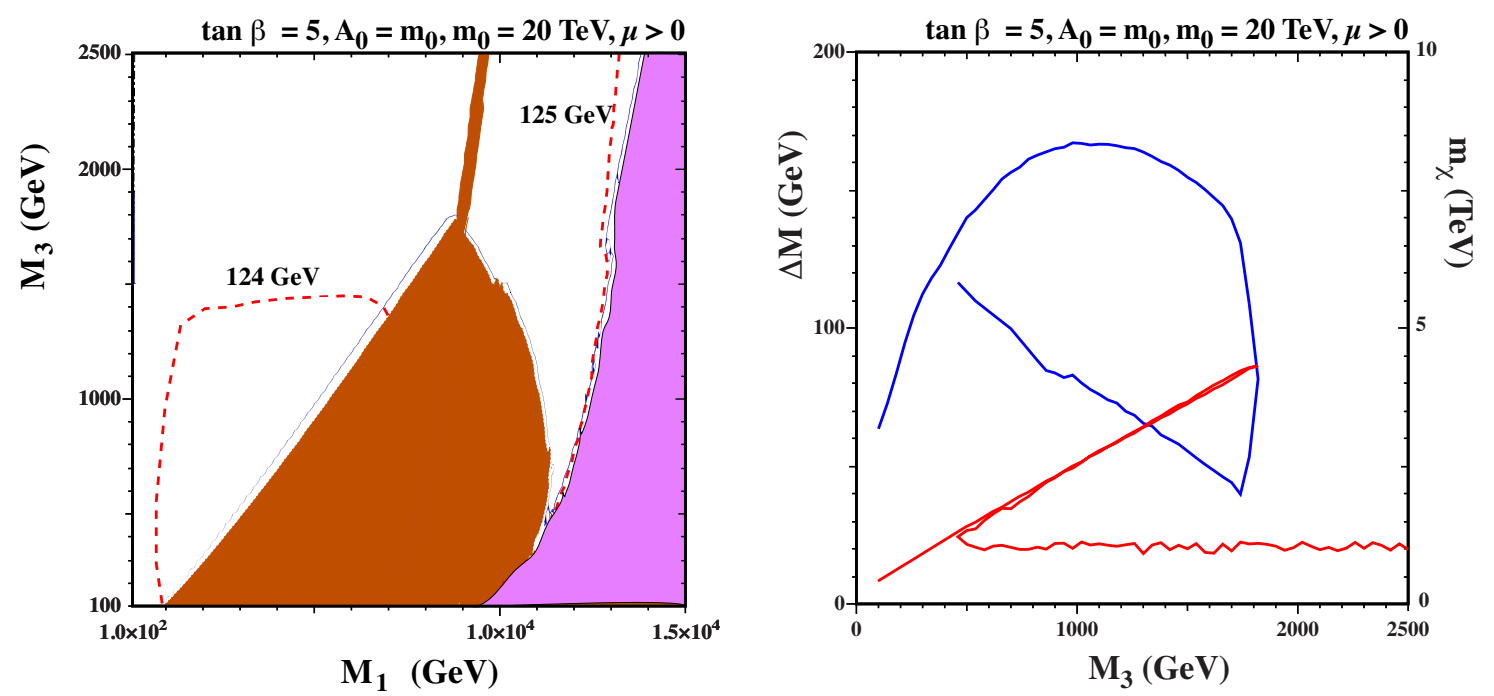

Figure 4. As for figure 1, but for $m_{0}=20 \mathrm{TeV}$ and $\tan \beta=5$ with $A_{0} / m_{0}=1.0$.

coannihilation strip extending nearly all the way to $M_{3} \sim 3 \mathrm{TeV}$. The panel on the right again shows the gluino-neutralino mass difference $\Delta M$ (blue line) which in this case peaks at approximately $170 \mathrm{GeV}$, which is consistent with the results of [72] for intermediate squark-to-gluino mass ratios. Also shown is the neutralino mass as a function of $M_{3}$ (red line): it again rises to $m_{\chi} \sim 8 \mathrm{TeV}$ at the tip of the coannihilation strip, which has $M_{3}$ slightly $>3 \mathrm{TeV}$. We list in the second column of table 1 some details of a sample parameter set from the model plane in figure 3 .

The Higgs mass in this case is again very slowly varying across the plane and takes the value $m_{H} \sim 125 \mathrm{GeV}$ for this choice of $\tan \beta=3$. In comparison, had we chosen $\tan \beta=5$, the $\left(M_{1}, M_{3}\right)$ plane would look almost identical but with $m_{H} \sim 131 \mathrm{GeV}$. This and larger values of $\tan \beta$ are therefore excluded for this value of $A_{0} / m_{0}$. We have also studied smaller values of $A_{0} / m_{0}$ and found no consistent solutions of the electroweak symmetry-breaking conditions for $M_{3} \lesssim 500 \mathrm{GeV}$ for $A_{0} / m_{0}=1$ and no consistent solutions across the plane at somewhat lower $A_{0} / m_{0}$. This is also the case for $\tan \beta=10$ and $A_{0} / m_{0}=1.5$, for which $m_{H} \sim 134 \mathrm{GeV}$. Larger values of $A_{0} / m_{0}$ also give values that tend to increase $m_{H}$ and, if increased too much, the stop becomes the LSP and eventually tachyonic.

Next we consider some sample $\left(M_{1}, M_{3}\right)$ planes with $m_{0}=20 \mathrm{TeV}$ and $\tan \beta=5$, corresponding to the lower end of the $m_{\chi}$ plateau in figure 2. Figure 4 is for the case $A_{0} / m_{0}=1$, where we see in the left panel that electroweak symmetry breaking is possible up to values of $M_{1} \lesssim 14 \mathrm{TeV}$. There is a gluino coannihilation strip close to the colored LSP boundary for $M_{1} \lesssim 9 \mathrm{TeV}$. This is terminated by a spur extending to large $M_{3}$ when $9 \mathrm{TeV}$ $\lesssim M_{1} \lesssim 10 \mathrm{TeV}$, where the lighter chargino is the LSP. There is no chargino coannihilation strip along the boundary of this region at large $M_{3}$, because the relic density is too high: for these values of $M_{1}$ and $M_{3}$, the Higgsino mass is too large and other coannihilations are not sufficient to bring the relic density down. At larger values, $10 \mathrm{TeV} \lesssim M_{1} \lesssim 11 \mathrm{TeV}$, there is a Higgsino-gluino coannihilation strip, which is followed at larger $M_{1}$ by a focus-point 
strip [116-121] hugging the electroweak symmetry breaking boundary where the neutralino is well-tempered [122]..$^{5}$ In this case we see both the 124 and $125 \mathrm{GeV}$ Higgs mass contours and, as in the previous example, $m_{H}$ is compatible with experiment whenever the dark matter density falls within the allowed range.

Because the relic density strip is a multi-valued function of $M_{3}$, the structure of the gluino-neutralino mass difference $\Delta M$ (blue curve) and the neutralino mass (red curve) shown in the right panel of figure 4 are more complicated than in the previous cases. After growing to a local maximum $\sim 170 \mathrm{GeV}$ when $M_{3} \sim 1 \mathrm{TeV}, \Delta M$ starts to fall at larger $M_{3}$. We then see a change in behaviour at $M_{3} \approx 1800 \mathrm{GeV}$ along the gluino coannihilation strip. Here, the neutralino becomes Higgsino-like and, as $M_{1}$ is increased, the coannihilation strip tends toward lower $M_{3}$ with an increasing mass difference, as seen in the lower branch of the blue curve. A Higgsino LSP emerges for larger $M_{1}$ because it gives a positive contribution to the up Higgs soft mass from renormalization group running. As the up Higgs soft mass goes to zero so does $\mu$ and the Higgsino becomes the LSP. Once $\mu$ is small enough, the Higgsino can be a thermal relic without any assistance in setting the relic density from other particles. In this focus-point-like region, the mass difference increases beyond the range displayed. This behaviour is correlated with the value of $m_{\chi}$ (red curve), which increases monotonically to $\sim 4 \mathrm{TeV}$. When the Bino/Higgsino transition occurs at $M_{3} \approx 1800 \mathrm{GeV}, m_{\chi}$ doubles back down to $M_{3} \approx 500 \mathrm{GeV}$. Then, on the focuspoint branch of the relic density strip, the LSP is mostly Higgsino, the value of $M_{3}$ grows, and the lightest neutralino mass takes the characteristic value $m_{\chi} \sim 1 \mathrm{TeV}$. We list in the third, fourth and fifth columns of table 1 some details of sample parameter sets from the model plane in figure 4.

In figure 5 we choose a larger value of $A_{0} / m_{0}=1.5$, and we see in the left panel a gluino coannihilation strip that extends to $M_{1} \sim 14 \mathrm{TeV}$, along which $m_{H}$ varies between 124 and $126 \mathrm{GeV}$ as seen by the three Higgs mass contours. The focus-point Higgsino dark matter region has disappeared, due to the large $A_{0}$ driving the Higgs mass to large negative values. The end-point of the gluino coannihilation strip is clearly seen in the right panel of figure 5 , where $\Delta M \rightarrow 0$ (blue curve) at $M_{3} \simeq 3300 \mathrm{GeV}$. Qualitatively, this case is similar to that shown in figure 3 , rather than to figure 4 with its truncated gluino coannihilation strip. In this case, the LSP mass (red curve) rises monotonically to $m_{\chi} \sim 7.5 \mathrm{TeV}$ at the end-point of the strip. We list in the sixth column of table 1 some details of a sample parameter set from the model plane in figure 5 .

In the left panel of figure 6 we display the $\left(M_{1}, M_{3}\right)$ plane for $m_{0}=20 \mathrm{TeV}$ and $A_{0} / m_{0}=2$. We see again a gluino coannihilation strip, but extending only to $M_{1} \sim$ $7.5 \mathrm{TeV}$. It is terminated by a stop LSP region that extends to larger values of $M_{3}$ than those displayed. In principle, one might have expected to see a stop coannihilation strip running up along the boundary of the stop LSP region. However, in this case the relic density is too high along the boundary shown in this figure: as in the chargino case mentioned earlier, the would-be end-point of the stop coannihilation strip lies within the gluino LSP region.

\footnotetext{
${ }^{5} \mathrm{~A}$ one-parameter extension of the CMSSM with lighter gluinos was considered in [123] in the context of a mixed Higgsino-Bino neutralino.
} 

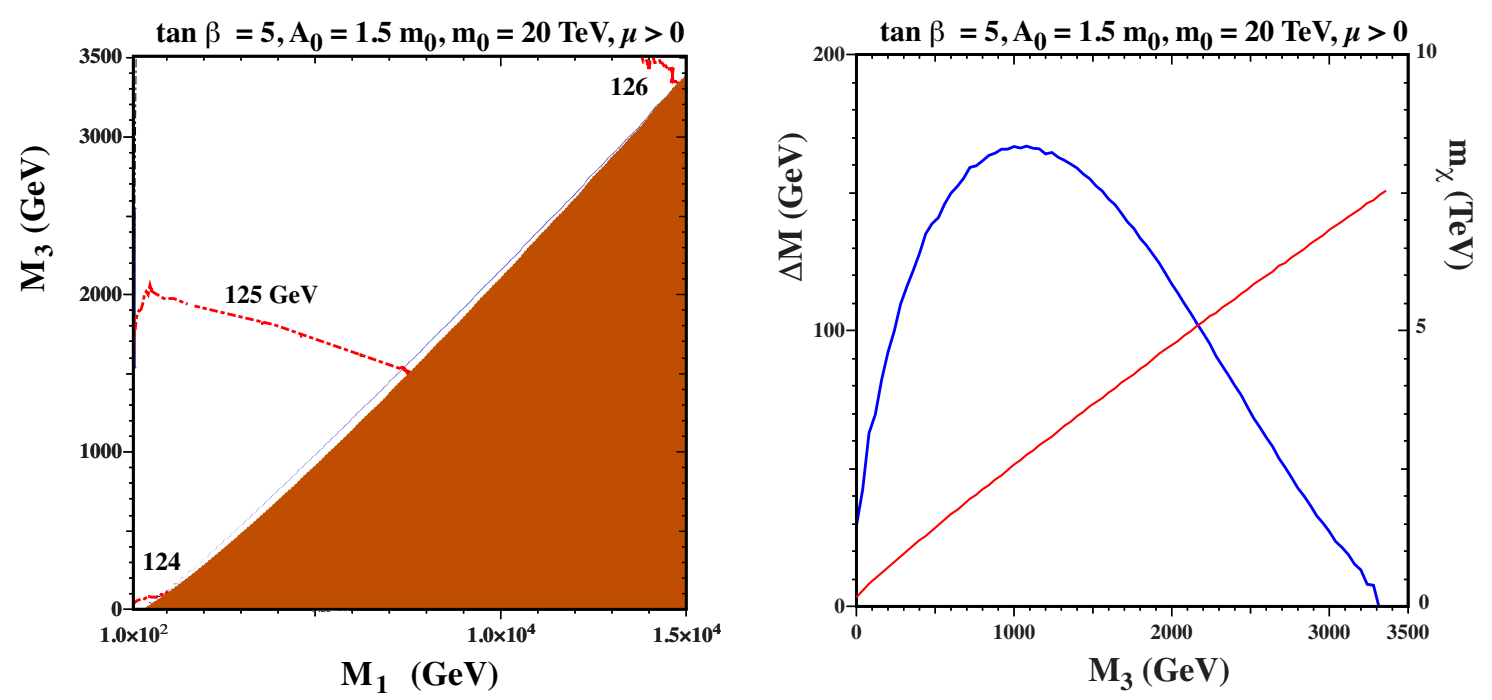

Figure 5. As for figure 1, but for $m_{0}=20 \mathrm{TeV}$ and $\tan \beta=5$ with $A_{0} / m_{0}=1.5$.
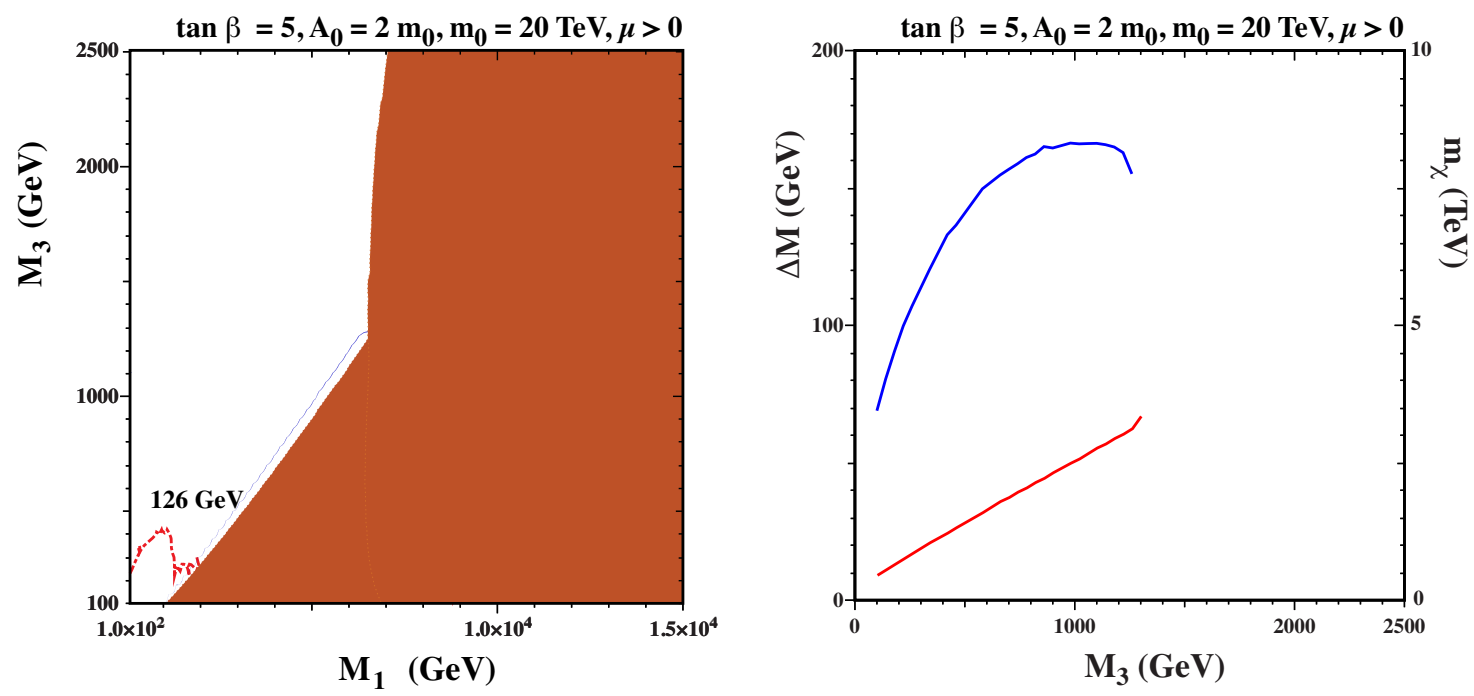

Figure 6. As for figure 1, but for $m_{0}=20 \mathrm{TeV}$ and $\tan \beta=5$ with $A_{0} / m_{0}=2.0$.

The value of $m_{H}$ is generally higher than in the previous case, though compatible with experiment along all the dark matter strip. In the right panel of figure 6 , the curves for $\Delta M$ and $m_{\chi}$ terminate when the stop becomes the LSP, with $m_{\chi} \lesssim 3.5 \mathrm{TeV}$. We list in the seventh column of table 1 some details of a sample parameter set from the model plane in figure 6 .

In figure 7 , we choose a lower value of $A_{0} / m_{0}=0.75$ and keep $\tan \beta=5$. We see, in the left panel, that consistent electroweak symmetry breaking is possible only for relatively large $M_{3}$ and small $M_{1}$, and that there is a strip hugging the curved electroweak symmetrybreaking boundary where the LSP has an enhanced Higgsino component. Its relic density is brought into the allowed range by the same mechanism as we discussed in the case of a 

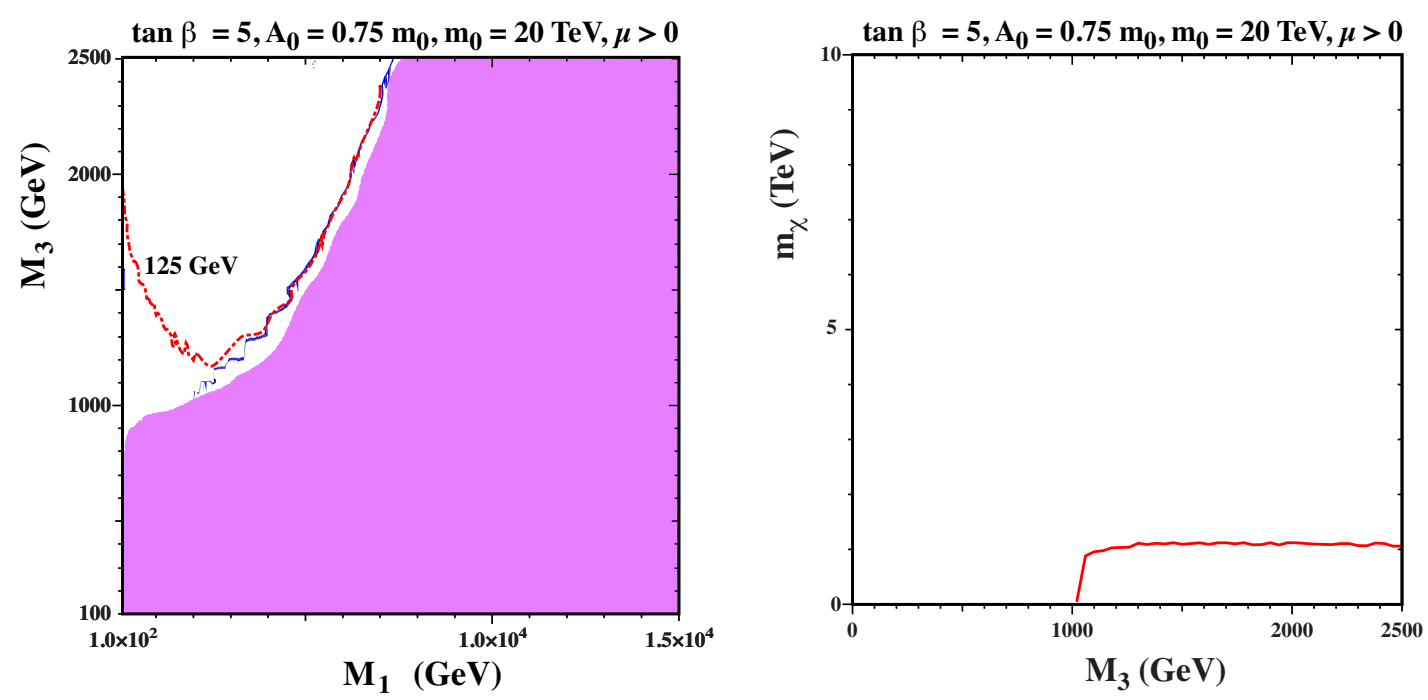

Figure 7. As for figure 1 , but for $m_{0}=20 \mathrm{TeV}$ and $\tan \beta=5$ with $A_{0} / m_{0}=0.75$.

well-tempered neutralino. As one can see in the right panel of figure 7 , once $M_{3}$ is large enough for EWSB solutions to exist, the mass of the lightest neutralino (which is mainly a Higgsino) is $m_{\chi} \sim 1.1 \mathrm{TeV}$ (red line), almost independent of $M_{3}$ for values $\gtrsim 1.1 \mathrm{TeV}$. The gluino-neutralino mass difference does not play a role in the relic density determination and is not shown here. The red dot-dashed contour shows where $m_{H}=125 \mathrm{GeV}: m_{H}$ is smaller (larger) above (below) this contour. The Higgs mass is highly compatible with the LHC measurement all along the displayed part of the relic density strip. We list in the eighth column of table 1 some details of a sample parameter set from the model plane in figure 7 .

At larger values of $\tan \beta$, the planes would look similar, though $m_{H}$ would be larger. The $\left(M_{1}, M_{3}\right)$ plane for $m_{0}=20 \mathrm{TeV}$ with $\tan \beta=10$ and $A_{0} / m_{0}=1$ resembles that in the left panel of figure 7 for $\tan \beta=5$ and $A_{0} / m_{0}=0.75$, with a focus-point strip following closely the curved electroweak symmetry breaking boundary. The most notable difference is the Higgs mass $m_{H}$, which is around $128 \mathrm{GeV}$ and only marginally compatible with experiment after allowing for the theoretical uncertainties. For the same values of $m_{0}$ and $\tan \beta=10$, we find no consistent electroweak symmetry breaking for smaller values of $A_{0} / m_{0}$, and for larger values we find that $m_{H}$ is too high. Thus we find no interesting examples of gluino coannihilation for $m_{0}=20 \mathrm{TeV}$ and $\tan \beta=10$.

Finally, we consider in figures 8 and 9 two examples for $m_{0}=10 \mathrm{TeV}$, corresponding to values of $m_{\tilde{q}} / m_{\chi}$ below the $m_{\chi}$ plateau in figure 2 . Figure 8 is for $\tan \beta=10$ and $A_{0} / m_{0}=1$ and displays a truncated gluino coannihilation strip extending to $M_{1} \sim 4 \mathrm{TeV}$, followed by a Higgsino coannihilation strip extending to $M_{1} \sim 5 \mathrm{TeV}$, and then a focus-point strip extending beyond the limits of the plot. We find that $m_{H}$ is always compatible with the experimental measurement. This example resembles that of figure 4 for $m_{0}=20 \mathrm{TeV}$ and $A_{0} / m_{0}=1$, the main difference being that the chargino spur has disappeared: we see instead a chargino LSP island at $M_{1} \sim 6 \mathrm{TeV}$ and $M_{3} \gtrsim 2 \mathrm{TeV}$. As in figure 4 , we see in 

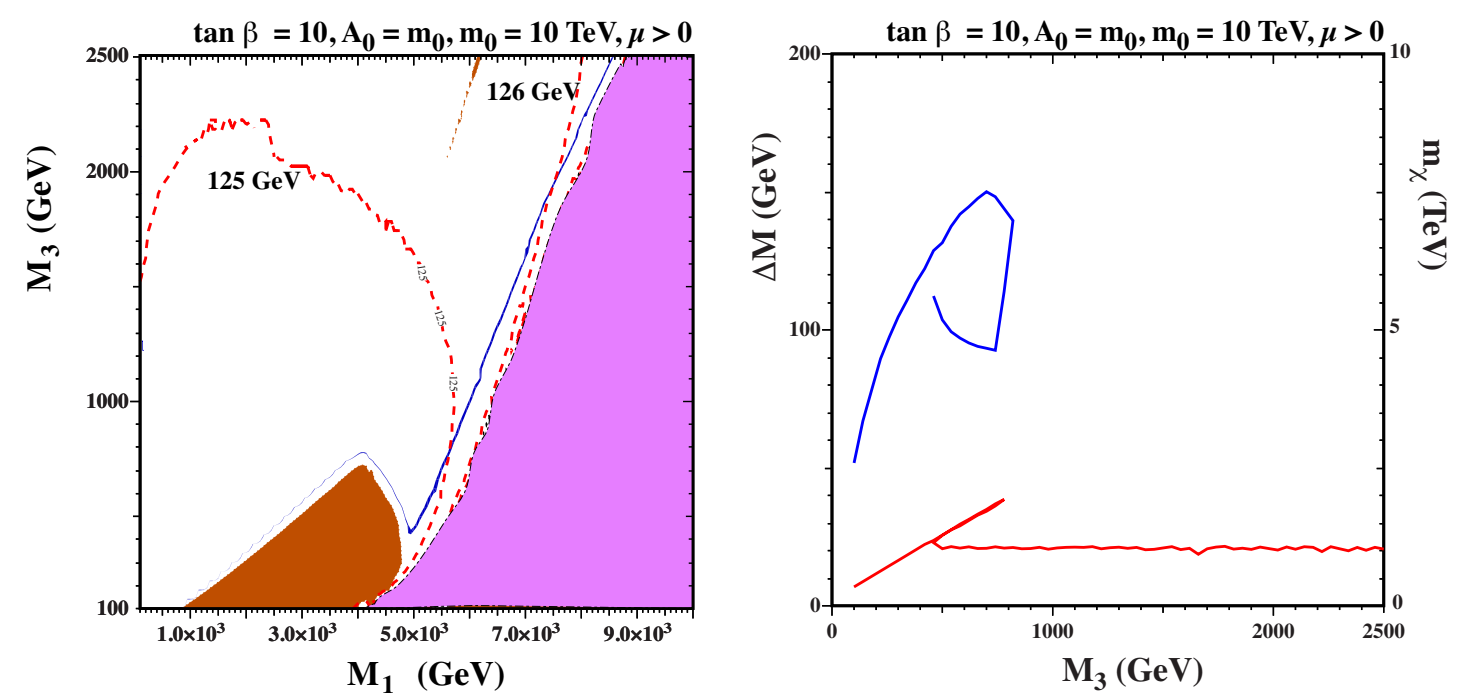

Figure 8. As for figure 1, but for $m_{0}=10 \mathrm{TeV}, \tan \beta=10$ and $A_{0} / m_{0}=1.0$.

the right panel that the gluino-neutralino mass difference (blue curve) has a multivalued form, and becomes larger as the neutralino becomes more Higgsino-like along the gluino coannihilation strip. At larger $M_{1}, M_{3}$ drops along the Higgsino-gluino coannihilation strip and the mass difference increases slightly as $M_{3}$ decreases, increasing beyond the displayed range as one moves on to the focus point strip. Similarly, the neutralino mass rises as $M_{1}$ is increased, then falls back to about $1.1 \mathrm{TeV}$ when the LSP is mostly a Higgsino. We list in the ninth, tenth and eleventh columns of table 1 some details of sample parameter sets from the model plane in figure 8.

Figure 9 for $A_{0} / m_{0}=1.5$ displays a more extended gluino coannihilation strip reaching $M_{1} \sim 7 \mathrm{TeV}$ and $m_{\chi} \sim 3.5 \mathrm{TeV}$, where it is terminated by a stop LSP region. This stop LSP region would dominate for larger values of $A_{0} / m_{0}$, and the range of $m_{H}$ would also become too high. At lower $\tan \beta$, the figures would look similar, but with a smaller Higgs mass. For example, for $\tan \beta=5$, with $A_{0} / m_{0}=1.5$ (as in figure 9 ), the Higgs mass would drop by roughly $3 \mathrm{GeV}$. Lower values of $\tan \beta$ would have $m_{H}$ too small, and lower values of $A_{0} / m_{0}$ but the same value of $\tan \beta$ would have no electroweak symmetry breaking solutions, while the stop LSP region would dominate for larger $A_{0} / m_{0}$. We list in the twelfth column of table 1 some details of a sample parameter set from the model plane in figure 9 .

Our analysis of gluino coannihilation in non-universal MSSM scenarios with $M_{1} \neq$ $M_{3}$ has shown that large values of $m_{\chi} \lesssim 8 \mathrm{TeV}$ are certainly possible, though restricted by competing mechanisms. This possibility occurs when $m_{0}=1000 \mathrm{TeV}$, but only for $A_{0} / m_{0} \sim 1.5$ and low values of $\tan \beta \lesssim 3$. The possibility of gluino coannihilation becomes more prominent for $m_{0}=200 \mathrm{TeV}$, appearing for an extended range of $\tan \beta$ though still only for $A_{0} / m_{0} \sim 1$.5. Gluino coannihilation is also prominent for $m_{0}=20 \mathrm{TeV}$ and $\tan \beta=5$, but the focus point may also be important (it becomes dominant when $\tan \beta=10$ ), as is Higgsino coannihilation. These features also appear when $m_{0}=10 \mathrm{TeV}$. In general, this scenario is constrained at small $A_{0} / m_{0}$ by the absence of electroweak symmetry breaking, and at large $A_{0} / m_{0}$ by $m_{H}$ and the appearance of a stop LSP. 

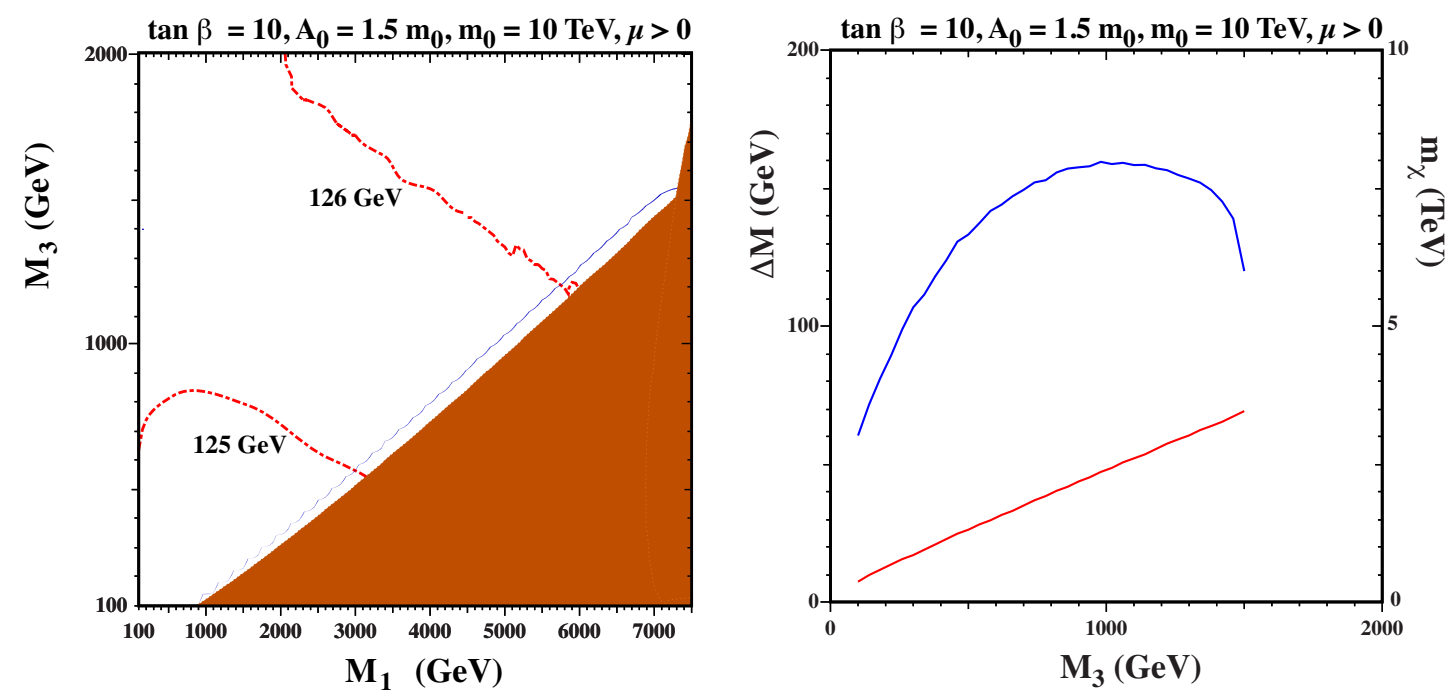

Figure 9. As for figure 1, but for $m_{0}=10 \mathrm{TeV}, \tan \beta=10$ and $A_{0} / m_{0}=1.5$.

\section{Pure gravity mediation with vector multiplets}

Another possible way of realizing a spectrum that can lead to gluino coannihilation is in models with pure gravity mediation [124-131] of supersymmetry breaking with additional vector multiplets that are charged under the SM gauge symmetries [67-69]. Since the mass spectrum of the gaugino sector of PGM is quite constrained, one of the few viable ways to alter this spectrum is through the addition of vector-like matter with all of its mass coming from a Giudice-Masiero (GM) term [132-134] in the Kähler potential. The vector-like matter alters the gaugino mass spectrum in two ways. First, it alters the beta functions of the SM gauge couplings that set the overall coefficient of the anomaly-mediated contribution to the gaugino masses. Secondly, when the additional vector-like matter is integrated out, it generates gauge-mediation-like contributions to the gaugino masses. ${ }^{6}$ Below we consider a PGM model with an additional pair of SU(5) $\mathbf{1 0}$ and $\overline{\mathbf{1 0}}$ multiplets, since this simple extension of PGM automatically generates a spectrum with gluino-Bino coannihilation.

The model we consider here is based on that in [69], whose setup we briefly review here. The effective potential is similar to that for the CMSSM:

$$
V=\left|\frac{\partial W}{\partial \phi^{i}}\right|^{2}+\left(A_{0} W^{(3)}+B_{0} W^{(2)}+\text { h.c. }\right)+m_{3 / 2}^{2} \phi^{i} \phi_{i}^{*},
$$

where $W^{(3)}$ corresponds to the trilinear terms of the superpotential, $W^{(2)}$ contains the bilinear terms of the superpotential, and the $\phi_{i}$ signify the MSSM fields, with

$$
W=\left(y_{e} H_{1} L e^{c}+y_{d} H_{1} Q d^{c}+y_{u} H_{2} Q u^{c}\right)+\mu H_{1} H_{2} .
$$

\footnotetext{
${ }^{6}$ If the masses of the vector-like states come from a term in the superpotential, when the vector multiplets are integrated out the gauge-mediated contribution to the mass exactly cancels the additional anomalymediated contribution, so that the anomaly-mediated relations are still valid below the mass of the additional vector states. If the mass comes from a GM term in the Kähler potential, this cancellation no longer occurs.
} 
The scalar masses are generated through gravity mediation with a minimal Kähler potential, in an identical manner to mSUGRA [135], and hence are equal to $m_{3 / 2}$ at the GUT scale. In general, as seen in (4.1), the form of $W^{(3)}$ dictates the pattern of the trilinear supersymmetry-breaking terms. However, the trilinear couplings are suppressed in PGM models, because the supersymmetry-breaking field is charged. Thus, we take $A_{0}=0$.

Since $A_{0}=0$ and the Kähler potential is minimal in this model, we have $B_{0}=-m_{3 / 2}$ for the Higgs fields. This leaves three free parameters, two of which are determined by enforcing the electroweak symmetry-breaking conditions:

$$
\mu^{2}=\frac{m_{1}^{2}-m_{2}^{2} \tan ^{2} \beta+\frac{1}{2} m_{Z}^{2}\left(1-\tan ^{2} \beta\right)+\Delta_{\mu}^{(1)}}{\tan ^{2} \beta-1+\Delta_{\mu}^{(2)}}
$$

and

$$
B \mu=-\frac{1}{2}\left(m_{1}^{2}+m_{2}^{2}+2 \mu^{2}\right) \sin 2 \beta+\Delta_{B}
$$

where $\Delta_{\mu}^{(1)}, \Delta_{\mu}^{(2)}$ and $\Delta_{B}$ are loop corrections to the relationships [136-138].

This scenario has a very restricted parameter space that does not, in general, realize electroweak symmetry breaking [131]. Therefore, we add a Giudice-Masiero (GM) term [132-134] for the Higgs fields, which modifies the GUT-scale values of both $B_{0}$ and $\mu$ :

$$
\begin{aligned}
\mu & =\mu_{0}+c_{H} m_{3 / 2}, \\
B \mu & =-\mu_{0} m_{3 / 2}+2 c_{H} m_{3 / 2}^{2} .
\end{aligned}
$$

This additional degree of freedom in the EWSB sector allows us to choose $\tan \beta$ (in addition to $\left.m_{3 / 2}\right)$ as a free parameter, and one finds viable parameter space as long as $\tan \beta \lesssim 3$ [131].

As already mentioned, the extension of this simplest viable version of the PGM scenario that we consider includes an additional $\mathbf{1 0}$ and $\overline{\mathbf{1 0}}$ of the SU(5) grand-unification group. Because these states are vector-like, the most general form of the Kähler potential is

$$
K=|\mathbf{1 0}|^{2}+|\overline{\mathbf{1 0}}|^{2}+\left(c_{10}(\mathbf{1 0} \cdot \overline{\mathbf{1 0}})+\text { h.c. }\right) \text {, }
$$

which includes a GM-like coupling $c_{10}$ that generates a supersymmetric mixing mass term, $\mu_{10}$, and a supersymmetry-breaking $B$ term for the additional vector-like fields. As can be seen from the minimal form of the kinetic terms in the limit $c_{10} \rightarrow 0$, the additional fields also have a gravity-mediated tree-level soft supersymmetry-breaking mass equal to $m_{3 / 2}$. Since some of the SM fields are also contained in a $\mathbf{1 0}$ of SU(5), the SM like fields contained in the additional $\mathbf{1 0}$ can be combined with $\mathrm{H}_{2}$, the $\mathrm{SU}(2)$ charged piece of the $\mathbf{5}_{H}$, into gauge-invariant operators in the superpotential just as is done in the SM. Since $H_{1}$ comes from the $\overline{\mathbf{5}}_{H}$, it can likewise be combined with the SM-charged components of the additional $\overline{\mathbf{1 0}}$ to form gauge-invariant Yukawa interactions. If we impose only gauge symmetries, the most generic contribution to the superpotential is

$$
W=y_{t}^{\prime} H_{u} Q^{\prime} U^{\prime}+y_{b}^{\prime} H_{d} \bar{Q} \bar{U}
$$


where $Q^{\prime}$ and $U^{\prime}$ are from the $\mathbf{1 0}$ and $\bar{Q}$ and $\bar{U}$ are from the $\overline{\mathbf{1 0}}$. However, to preserve $R$ symmetry $^{7}$ we must take either $y_{b}^{\prime}=0$ or $y_{t}^{\prime}=0$. Here, we take $y_{b}^{\prime}=0$. The interactions proportional to $y_{t}^{\prime}$ contribute to the beta function of the up Higgs soft mass in a similar way to those controlled by $y_{t}$. Specifying a comparable value of $y_{t}^{\prime}$ helps drive radiative electroweak symmetry breaking, which in turn allows larger values of $\tan \beta>3$. The extended theory now has four parameters: $m_{3 / 2}, \tan \beta, c_{10}$, and $y_{t}^{\prime}$.

The gaugino masses in these models are generated by anomalies [140-144]. Because the contributions to gaugino masses are proportional to $m_{3 / 2}$ times the gauge coupling beta function, the scalar masses tend to be much heavier than the gaugino masses, reminiscent of split supersymmetry [145-149]. With the addition of the $\mathbf{1 0}$ and $\overline{\mathbf{1 0}}$, the anomaly-mediated contributions to the gaugino masses are $^{8}$

$$
\begin{aligned}
M_{1} & =\frac{48}{5} \frac{g_{1}^{2}}{16 \pi^{2}} m_{3 / 2}, \\
M_{2} & =\frac{g_{2}^{2}}{4 \pi^{2}} m_{3 / 2}, \\
M_{3} & =0 .
\end{aligned}
$$

In addition, the gauginos then get rather large threshold corrections from the $\mathbf{1 0}$ and $\overline{\mathbf{1 0}}$ when they are integrated out, which is in addition to the large threshold correction coming from integrating out the Higgsinos: for more details see [69]. Since the only contribution to the mass of the gluino comes from the threshold corrections, it tends to be lighter than in typical PGM models. Hence there are regions where the gluino can coannihilate with the Bino, yielding the possibility of a relatively heavy Bino dark matter candidate.

Our results for the PGM model with vector $\mathbf{1 0}$ and $\overline{\mathbf{1 0}}$ multiplets can be displayed in $\left(c_{10}, m_{3 / 2}\right)$ planes for fixed values of the Yukawa coupling, $y_{t}^{\prime}$ and $\tan \beta$. Two examples of these planes are shown in figures 10 and 11. In the former, we have fixed $\tan \beta=3$ (mainly to get an acceptable value for the Higgs mass, $m_{H}$, over the range of $m_{3 / 2} \leq 600 \mathrm{TeV}$ shown) and $y_{t}^{\prime 2}=0.15$. In the left panel, we see a large red shaded region at small $c_{10}$ where the gluino is the LSP. To the right of this boundary, we see the gluino coannihilation strip. ${ }^{9}$ In the lower right corner, the pink shaded region is excluded because one or more of the scalar components of the new vector matter have become tachyonic. As in previous figures, the Higgs mass contours are shown as red dot-dashed curves as labelled. Within the theoretical uncertainties, the Higgs mass agrees with experiment over the part of the

\footnotetext{
${ }^{7}$ We recall that $R$ symmetry is an important part of dynamical supersymmetry breaking [139]. Since we wish ultimately to have supersymmetry broken dynamically, we consider a theory that preserves $R$ symmetry. We note furthermore that this $R$ symmetry can also play an important part in generating the PGM spectrum, since $R$ symmetry can forbid the tree-level gaugino masses generated in supergravity.

${ }^{8}$ The anomaly mediated gaugino masses are proportional to the gauge coupling beta functions. The addition of a $\mathbf{1 0}$ and $\overline{\mathbf{1 0}}$ alters the gauge coupling beta functions and so alters the gaugino masses. As it turns out, the additional contribution of the $\mathbf{1 0}$ and $\overline{\mathbf{1 0}}$ to the SU(3) beta function completely cancels the SM contribution at one-loop. Therefore, the total anomaly mediated contribution to the gluino mass is zero at one-loop.

${ }^{9}$ The strip becomes less well defined at $m_{3 / 2} \gtrsim 350 \mathrm{TeV}$ due to inaccuracies of the relic density calculation at such large masses.
} 

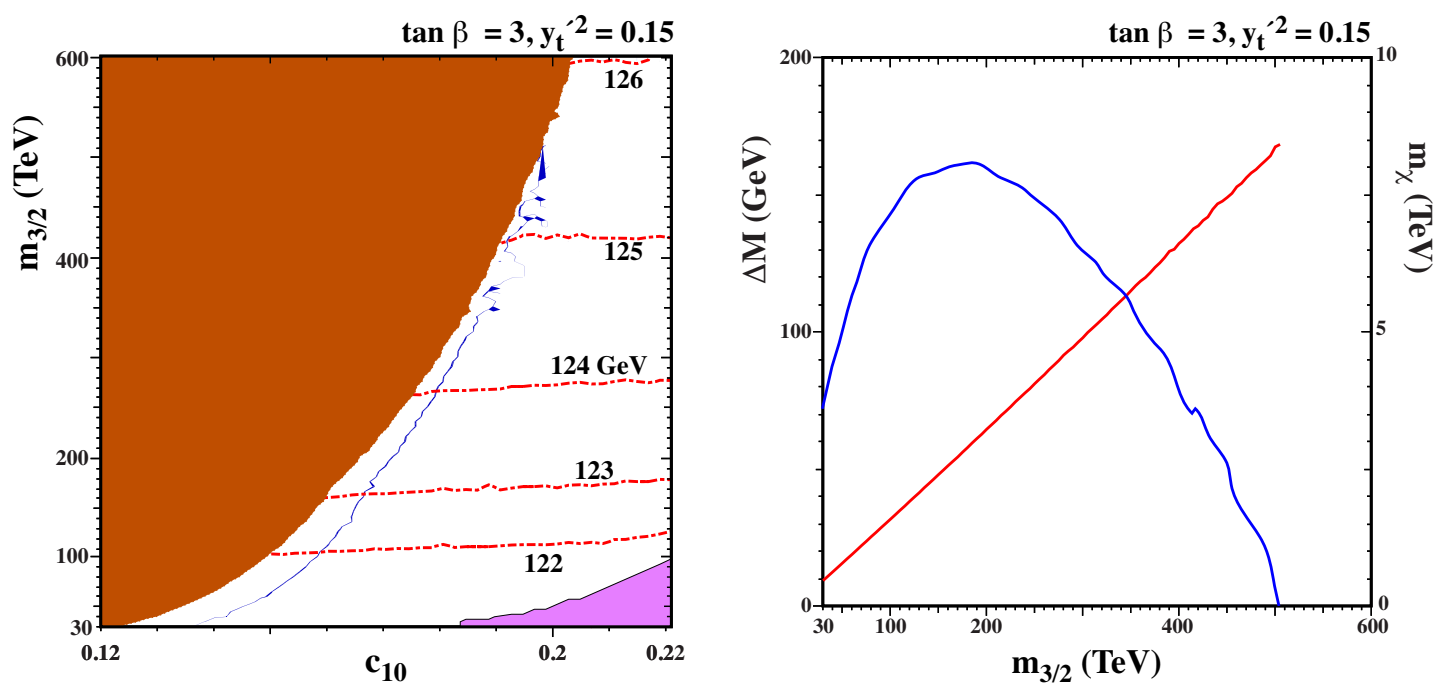

Figure 10. The PGM $\left(c_{10}, m_{3 / 2}\right)$ plane for fixed $\tan \beta=3$ and $y_{t}^{\prime 2}=0.15$. The dark blue strip in the left panel shows where the relic LSP density $\Omega_{\chi} h^{2}$ falls within the $\pm 3-\sigma$ range allowed by Planck and other data, and the lightest neutralino is no longer the LSP in the low- $c_{10}$ regions shaded brick-red. One or more of the new vector scalars becomes tachyonic in the lower right corner of the plane (shaded pink). The right panel shows the gluino-neutralino mass difference (left axis, blue line) and the neutralino mass (right axis, red line) as functions of $m_{3 / 2}$.

plane that is shown. In the right panel we see, as before, the gluino-neutralino mass difference $\Delta M$ along the gluino coannihilation strip (blue) and the neutralino mass along the strip (red). We see that the curve for $\Delta M$ has the same characteristic shape due to strong coannihilations involving the gluino and peaks at $\simeq 170 \mathrm{GeV}$ at $m_{3 / 2} \simeq 200 \mathrm{TeV}$ when $m_{\chi} \simeq 3 \mathrm{TeV}$. The end-point of the coannihilation strip occurs at $m_{3 / 2} \simeq 500 \mathrm{TeV}$ where $m_{\chi} \simeq 8.3 \mathrm{TeV} .{ }^{10}$ We list in the last two columns of table 1 some details of sample parameter sets from the model planes in figures 10 and 11 . We recall that $A_{0}=0$ in these models, and display in parentheses $(\ldots)$ the model values of $c_{10}$.

In figure 11 , we show in the left panel the corresponding $\left(c_{10}, m_{3 / 2}\right)$ plane for fixed $y_{t}^{\prime 2}=0.65$ and the same value of $\tan \beta=3$. In this case with a higher Yukawa coupling, slightly higher $c_{10}$ is needed to obtain a neutralino LSP. As in the previous case, we see a brick-red shaded gluino LSP for low $c_{10}$ and, at slightly larger $c_{10}$, a gluino coannihilation strip. As previously, $m_{H}$ is acceptable along all the displayed portion of the strip where $m_{3 / 2} \lesssim 400 \mathrm{TeV}$. In this case, the region at larger $c_{10}$ where one or more of the new vector scalars becomes tachyonic also extends to low $c_{10}$ for small $m_{3 / 2}$. The right panel of figure 11 shows the values of $\Delta M$ and $m_{\chi}$ along the gluino coannihilation strip. In this case, we see that $\Delta M$ is maximized at $\sim 160 \mathrm{GeV}$ for $m_{3 / 2} \sim 150 \mathrm{TeV}$. The end-point of the gluino coannihilation strip also occurs at $m_{3 / 2} \simeq 500 \mathrm{TeV}$ with $m_{\chi}$ around $8.3 \mathrm{TeV}$.

Finally, we show in figure 12 an example of a $\left(y_{t}^{\prime 2}, c_{10}\right)$ plane with $\tan \beta=5$ for four choices of the gravitino mass, namely $m_{3 / 2}=30,50,100$ and $250 \mathrm{TeV}$. The red shaded

\footnotetext{
${ }^{10}$ The maximal value of the LSP mass that is compatible with it being a viable dark matter candidate in this case is similar to the CMSSM case, even though there are additional squarks to mediate $\chi q \rightarrow q \tilde{g}$. This is because the limiting reaction is gluino-gluino annihilation, which is the same in the two cases.
} 

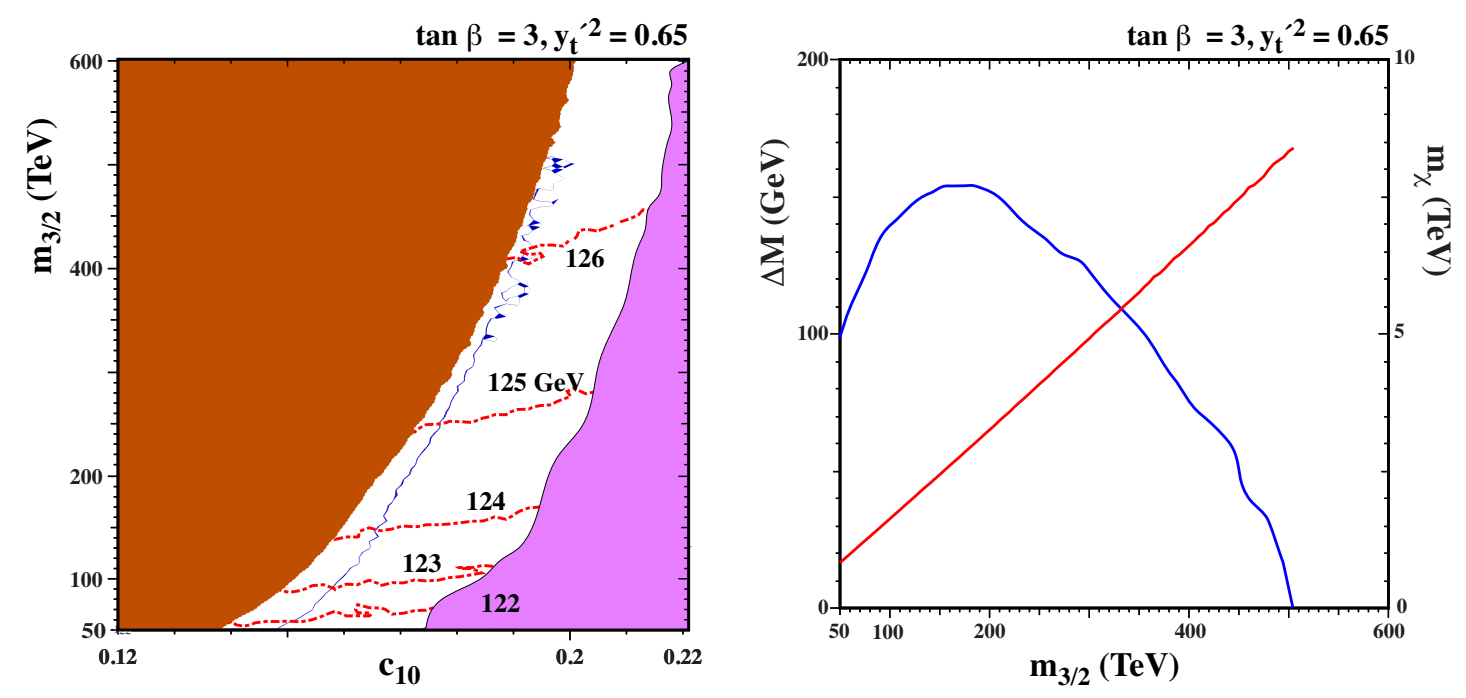

Figure 11. As in figure 10, the PGM $\left(c_{10}, m_{3 / 2}\right)$ plane for fixed $\tan \beta=3$ and $y_{t}^{\prime 2}=0.65$.

region has a gluino LSP only in the $m_{3 / 2}=30 \mathrm{TeV}$ case. In the other three cases this region would be displaced to larger $c_{10}$. Because the Higgs mass depends on $m_{3 / 2}$, there are no unique contours that can be displayed for all four cases. Instead, we have colorcoded the gluino coannihilation strip according to the Higgs mass: $124-125 \mathrm{GeV}$ (black), 125-126 GeV (blue), 126-127 GeV (green), $127-128 \mathrm{GeV}$ (red), and > $128 \mathrm{GeV}$ (yellow). ${ }^{11}$ The right panel shows that the gluino-neutralino mass difference is almost independent of $y_{t}^{\prime 2}$. We do not show the neutralino mass for these cases, as it is largely independent of $y_{t}^{\prime}$ and is determined from the gravitino mass and can be read from either of the two previous figures.

\section{$5 \quad$ Summary and conclusions}

We have presented in this paper a couple of representative MSSM scenarios in which the gluino may be nearly degenerate with the neutralino LSP $\chi$, whose relic dark matter density is brought into the range favoured by Planck and other data by gluino coannihilation. It had been shown previously that values of $m_{\chi} \lesssim 8 \mathrm{TeV}$ are in principle possible when gluino coannihilation is operative [72], and we have shown in this paper how such a possibility can be embedded within a scenario for non-universal soft supersymmetry breaking within the MSSM, on the one hand, and within a simple extension of the MSSM with pure gravity mediation of soft supersymmetry breaking that includes a vector-like $\mathbf{1 0}+\overline{\mathbf{1 0}}$ multiplet pair, on the other hand.

In both scenarios, the upper bound on $m_{\chi}$ depends on the details of the models. In particular, in the non-universal MSSM scenario there is competition from other mechanisms for bringing the dark matter density into the Planck range. These, together with other phenomenological constraints such as the mass of the Higgs boson and the requirement to

\footnotetext{
${ }^{11}$ We recall also that the Higgs mass is sensitive to the choice of $\tan \beta$.
} 

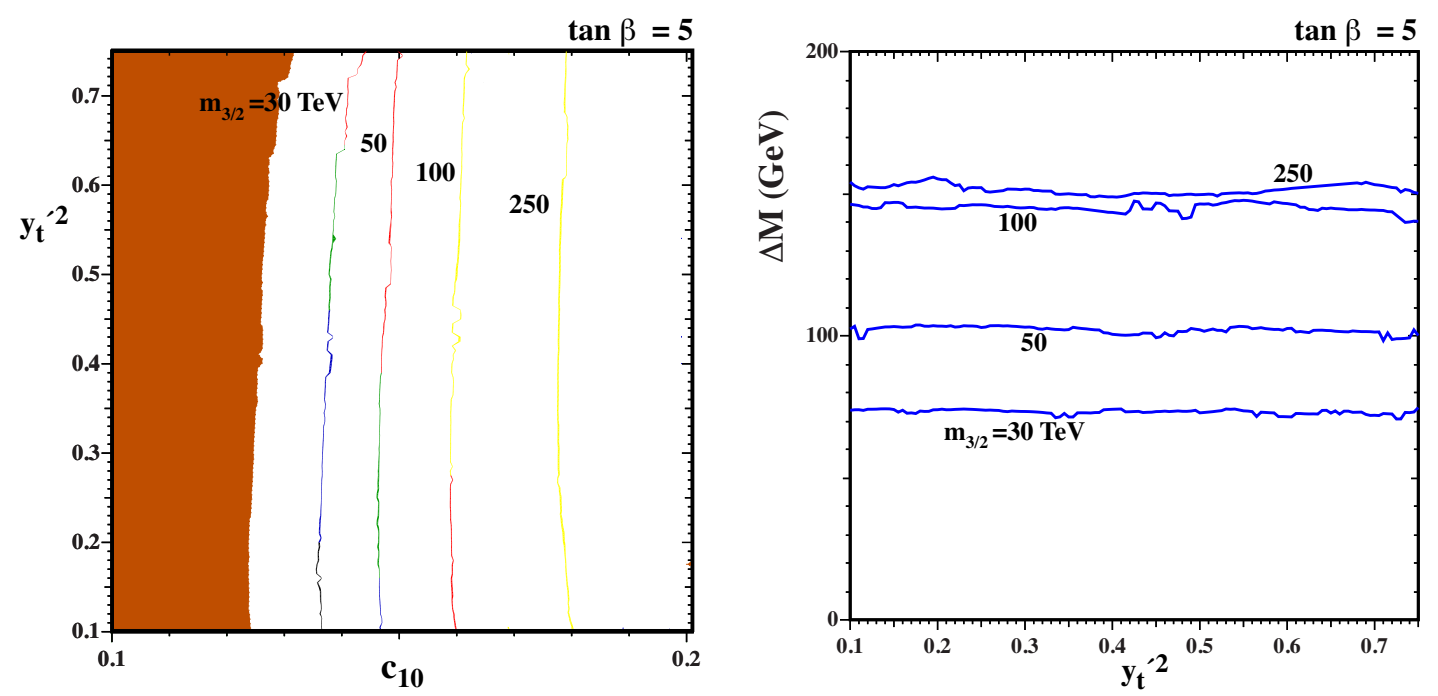

Figure 12. The PGM $\left(y_{t}^{\prime 2}, c_{10}\right)$ plane with $\tan \beta=5$ for four choices of the gravitino mass, $m_{3 / 2}=30,50,100$ and $250 \mathrm{TeV}$. The right panel shows the gluino-neutralino mass difference as a function of $y_{t}^{\prime 2}$.

ensure electroweak symmetry breaking, restrict the parameter region where gluino coannihilation is dominant. In the PGM scenario with extra vector-like multiplets, the allowed range of $m_{\chi}$ depends on the gravitino mass as well as a vector-like Yukawa coupling. In both cases, values of $m_{\chi} \sim 8 \mathrm{TeV}$ are quite possible.

Gluino coannihilation therefore offers the possibility that the LSP, and hence the rest of the supersymmetric spectrum, may lie in the multi-TeV range, beyond the reach of the LHC. Of course, we sincerely hope, if not expect, that supersymmetry will be discovered during future LHC runs. That said, the scenarios discussed here illustrate one way in which detection at the LHC could be evaded. An interesting and important question that lies beyond the scope of this paper is how to detect supersymmetry in a gluino coannihilation scenario with a multi-TeV LSP (see, e.g., the discussion in [150]). As we have discussed in this paper, the gluino-neutralino mass difference in such a scenario is typically $\mathcal{O}(100) \mathrm{GeV}$, resulting in a suppressed missing-energy signature whose detection at a future $100-\mathrm{TeV}$ proton-proton collider might be challenging.

Une affaire à suivre.

\section{Acknowledgments}

The work of J.E. was supported in part by the London Centre for Terauniverse Studies (LCTS), using funding from the European Research Council via the Advanced Investigator Grant 267352 and from the U.K. STFC via the research grant ST/J002798/1. The work of F.L. was also supported by the European Research Council Advanced Investigator Grant 267352. The work of J.L.E. and K.A.O. was supported in part by DOE grant DE-SC0011842 at the University of Minnesota. 
Open Access. This article is distributed under the terms of the Creative Commons Attribution License (CC-BY 4.0), which permits any use, distribution and reproduction in any medium, provided the original author(s) and source are credited.

\section{References}

[1] ATLAS collaboration, Search for squarks and gluinos with the ATLAS detector in final states with jets and missing transverse momentum using $\sqrt{s}=8$ TeV proton-proton collision data, JHEP 09 (2014) 176 [arXiv:1405.7875] [INSPIRE].

[2] ATLAS collaboration, Summary of the searches for squarks and gluinos using $\sqrt{s}=8 \mathrm{TeV}$ pp collisions with the ATLAS experiment at the LHC, JHEP 10 (2015) 054 [arXiv: 1507.05525] [INSPIRE].

[3] CMS collaboration, Search for new physics in the multijet and missing transverse momentum final state in proton-proton collisions at $\sqrt{s}=8 \mathrm{TeV}$, JHEP 06 (2014) 055 [arXiv:1402.4770] [INSPIRE].

[4] ATLAS collaboration, A search for R-parity violating scalar top decays in all-hadronic final states with the ATLAS detector in $\sqrt{s}=8 \mathrm{TeV}$ pp collisions, ATLAS-CONF-2015-026 (2015) [INSPIRE].

[5] CMS collaboration, Search for R-parity-violating supersymmetry in proton-proton collisions at $\sqrt{s}=8 \mathrm{TeV}$ in events with large jet and b-jet multiplicity, CMS-PAS-SUS-14-003 (2015) [INSPIRE].

[6] CMS collaboration, Search for dark matter and compressed mass-spectra supersymmetry with the vector boson fusion topology in pp collisions at $\sqrt{s}=8 \mathrm{TeV}$, CMS-PAS-SUS-14-019 (2015) [INSPIRE].

[7] S.P. Martin, Compressed supersymmetry and natural neutralino dark matter from top squark-mediated annihilation to top quarks, Phys. Rev. D 75 (2007) 115005 [hep-ph/0703097] [INSPIRE].

[8] B. Bhattacherjee, A. Choudhury, K. Ghosh and S. Poddar, Compressed supersymmetry at $14 \mathrm{TeV}$ LHC, Phys. Rev. D 89 (2014) 037702 [arXiv:1308.1526] [InSPIRE].

[9] G. Chalons and D. Sengupta, Closing in on compressed gluino-neutralino spectra at the LHC, JHEP 12 (2015) 129 [arXiv: 1508.06735] [INSPIRE].

[10] O. Buchmueller et al., Collider Interplay for Supersymmetry, Higgs and Dark Matter, Eur. Phys. J. C 75 (2015) 469 [arXiv: 1505.04702] [InSPIRE].

[11] H. Goldberg, Constraint on the Photino Mass from Cosmology, Phys. Rev. Lett. 50 (1983) 1419 [Erratum ibid. 103 (2009) 099905] [INSPIRE].

[12] J.R. Ellis, J.S. Hagelin, D.V. Nanopoulos, K.A. Olive and M. Srednicki, Supersymmetric Relics from the Big Bang, Nucl. Phys. B 238 (1984) 453 [INSPIRE].

[13] Planck collaboration, P.A.R. Ade et al., Planck 2015 results. XIII. Cosmological parameters, arXiv:1502.01589 [INSPIRE].

[14] J.R. Ellis and K.A. Olive, Revisiting the Higgs Mass and Dark Matter in the CMSSM, Eur. Phys. J. C 72 (2012) 2005 [arXiv:1202.3262] [INSPIRE].

[15] J.R. Ellis, F. Luo, K.A. Olive and P. Sandick, The Higgs Mass beyond the CMSSM, Eur. Phys. J. C 73 (2013) 2403 [arXiv:1212.4476] [INSPIRE]. 
[16] J.R. Ellis, J.L. Evans, F. Luo, N. Nagata, K.A. Olive and P. Sandick, Beyond the CMSSM without an Accelerator: Proton Decay and Direct Dark Matter Detection, Eur. Phys. J. C 76 (2016) 8 [arXiv: 1509.08838] [INSPIRE].

[17] E.A. Bagnaschi et al., Supersymmetric Dark Matter after LHC Run 1, Eur. Phys. J. C 75 (2015) 500 [arXiv: 1508.01173] [INSPIRE].

[18] L. Aparicio, D.G. Cerdeno and L.E. Ibáñez, A 119-125 GeV Higgs from a string derived slice of the CMSSM, JHEP 04 (2012) 126 [arXiv:1202.0822] [INSPIRE].

[19] H. Baer, V. Barger and A. Mustafayev, Neutralino dark matter in mSUGRA/CMSSM with a $125 \mathrm{GeV}$ light Higgs scalar, JHEP 05 (2012) 091 [arXiv:1202.4038] [INSPIRE].

[20] P. Bechtle et al., Constrained Supersymmetry after two years of LHC data: a global view with Fittino, JHEP 06 (2012) 098 [arXiv: 1204.4199] [inSPIRE].

[21] C. Balázs, A. Buckley, D. Carter, B. Farmer and M. White, Should we still believe in constrained supersymmetry?, Eur. Phys. J. C 73 (2013) 2563 [arXiv:1205.1568] [InSPIRE].

[22] D. Ghosh, M. Guchait, S. Raychaudhuri and D. Sengupta, How Constrained is the cMSSM?, Phys. Rev. D 86 (2012) 055007 [arXiv: 1205. 2283] [inSPIRE].

[23] A. Fowlie et al., The CMSSM Favoring New Territories: The Impact of New LHC Limits and a $125 \mathrm{GeV}$ Higgs, Phys. Rev. D 86 (2012) 075010 [arXiv: 1206. 0264] [InSPIRE].

[24] K. Kowalska, S. Munir, L. Roszkowski, E.M. Sessolo, S. Trojanowski and Y.-L.S. Tsai, Constrained next-to-minimal supersymmetric standard model with a $126 \mathrm{GeV}$ Higgs boson: A global analysis, Phys. Rev. D 87 (2013) 115010 [arXiv:1211.1693] [InSPIRE].

[25] C. Strege, G. Bertone, F. Feroz, M. Fornasa, R. Ruiz de Austri and R. Trotta, Global Fits of the CMSSM and NUHM including the LHC Higgs discovery and new XENON100 constraints, JCAP 04 (2013) 013 [arXiv: 1212.2636] [INSPIRE].

[26] M.E. Cabrera, J.A. Casas and R. Ruiz de Austri, The health of SUSY after the Higgs discovery and the XENON100 data, JHEP 07 (2013) 182 [arXiv:1212.4821] [INSPIRE].

[27] T. Cohen and J.G. Wacker, Here be Dragons: The Unexplored Continents of the CMSSM, JHEP 09 (2013) 061 [arXiv: 1305.2914] [INSPIRE].

[28] S. Henrot-Versillé et al., Constraining Supersymmetry using the relic density and the Higgs boson, Phys. Rev. D 89 (2014) 055017 [arXiv: 1309.6958] [INSPIRE].

[29] P. Bechtle et al., Constrained Supersymmetry after the Higgs Boson Discovery: A global analysis with Fittino, PoS (EPS-HEP 2013) 313 [arXiv:1310.3045] [INSPIRE].

[30] L. Roszkowski, E.M. Sessolo and A.J. Williams, What next for the CMSSM and the NUHM: Improved prospects for superpartner and dark matter detection, JHEP 08 (2014) 067 [arXiv: 1405.4289] [INSPIRE].

[31] P. Bechtle et al., Killing the CMSSM softly, arXiv:1508.05951 [INSPIRE].

[32] M. Drees and M.M. Nojiri, The Neutralino relic density in minimal $N=1$ supergravity, Phys. Rev. D 47 (1993) 376 [hep-ph/9207234] [InSPIRE].

[33] H. Baer and M. Brhlik, Cosmological relic density from minimal supergravity with implications for collider physics, Phys. Rev. D 53 (1996) 597 [hep-ph/9508321] [InSPIRE].

[34] H. Baer and M. Brhlik, Neutralino dark matter in minimal supergravity: Direct detection versus collider searches, Phys. Rev. D 57 (1998) 567 [hep-ph/9706509] [INSPIRE].

[35] H. Baer et al., Yukawa unified supersymmetric $\mathrm{SO}(10)$ model: Cosmology, rare decays and collider searches, Phys. Rev. D 63 (2000) 015007 [hep-ph/0005027] [INSPIRE]. 
[36] J.R. Ellis, T. Falk, G. Ganis, K.A. Olive and M. Srednicki, The CMSSM parameter space at large $\tan \beta$, Phys. Lett. B 510 (2001) 236 [hep-ph/0102098] [INSPIRE].

[37] K. Griest and D. Seckel, Three exceptions in the calculation of relic abundances, Phys. Rev. D 43 (1991) 3191 [INSPIRE].

[38] J.R. Ellis, T. Falk and K.A. Olive, Neutralino-stau coannihilation and the cosmological upper limit on the mass of the lightest supersymmetric particle, Phys. Lett. B 444 (1998) 367 [hep-ph/9810360] [INSPIRE].

[39] J.R. Ellis, T. Falk, K.A. Olive and M. Srednicki, Calculations of neutralino-stau coannihilation channels and the cosmologically relevant region of MSSM parameter space, Astropart. Phys. 13 (2000) 181 [Erratum ibid. 15 (2001) 413] [hep-ph/9905481] [InSPIRE].

[40] R.L. Arnowitt, B. Dutta and Y. Santoso, Coannihilation effects in supergravity and D-brane models, Nucl. Phys. B 606 (2001) 59 [hep-ph/0102181] [InSPIRE].

[41] M.E. Gómez, G. Lazarides and C. Pallis, Supersymmetric cold dark matter with Yukawa unification, Phys. Rev. D 61 (2000) 123512 [hep-ph/9907261] [INSPIRE].

[42] M.E. Gómez, G. Lazarides and C. Pallis, Yukawa unification, $b \rightarrow s \gamma$ and Bino-Stau coannihilation, Phys. Lett. B 487 (2000) 313 [hep-ph/0004028] [InSPIRE].

[43] M.E. Gómez, G. Lazarides and C. Pallis, Yukawa quasi-unification, Nucl. Phys. B 638 (2002) 165 [hep-ph/0203131] [INSPIRE].

[44] T. Nihei, L. Roszkowski and R. Ruiz de Austri, Exact cross-sections for the neutralino slepton coannihilation, JHEP 07 (2002) 024 [hep-ph/0206266] [INSPIRE].

[45] M. Citron, J.R. Ellis, F. Luo, J. Marrouche, K.A. Olive and K.J. de Vries, End of the CMSSM coannihilation strip is nigh, Phys. Rev. D 87 (2013) 036012 [arXiv:1212.2886] [INSPIRE].

[46] C. Boehm, A. Djouadi and M. Drees, Light scalar top quarks and supersymmetric dark matter, Phys. Rev. D 62 (2000) 035012 [hep-ph/9911496] [INSPIRE].

[47] J.R. Ellis, K.A. Olive and Y. Santoso, Calculations of neutralino stop coannihilation in the CMSSM, Astropart. Phys. 18 (2003) 395 [hep-ph/0112113] [INSPIRE].

[48] J.L. Diaz-Cruz, J.R. Ellis, K.A. Olive and Y. Santoso, On the Feasibility of a Stop NLSP in Gravitino Dark Matter Scenarios, JHEP 05 (2007) 003 [hep-ph/0701229] [INSPIRE].

[49] I. Gogoladze, S. Raza and Q. Shafi, Light stop from $b-\tau$ Yukawa unification, Phys. Lett. B 706 (2012) 345 [arXiv:1104.3566] [INSPIRE].

[50] M.A. Ajaib, T. Li and Q. Shafi, Stop-Neutralino Coannihilation in the Light of LHC, Phys. Rev. D 85 (2012) 055021 [arXiv: 1111.4467] [INSPIRE].

[51] J. Harz, B. Herrmann, M. Klasen, K. Kovarik and Q.L. Boulc'h, Neutralino-stop coannihilation into electroweak gauge and Higgs bosons at one loop, Phys. Rev. D 87 (2013) 054031 [arXiv: 1212.5241] [INSPIRE].

[52] J. Harz, B. Herrmann, M. Klasen and K. Kovarik, One-loop corrections to neutralino-stop coannihilation revisited, Phys. Rev. D 91 (2015) 034028 [arXiv:1409.2898] [INSPIRE].

[53] J.R. Ellis, K.A. Olive and J. Zheng, The Extent of the Stop Coannihilation Strip, Eur. Phys. J. C 74 (2014) 2947 [arXiv: 1404.5571] [INSPIRE].

[54] A. Ibarra, A. Pierce, N.R. Shah and S. Vogl, Anatomy of Coannihilation with a Scalar Top Partner, Phys. Rev. D 91 (2015) 095018 [arXiv:1501.03164] [INSPIRE]. 
[55] J. Edsjo, M. Schelke, P. Ullio and P. Gondolo, Accurate relic densities with neutralino, chargino and sfermion coannihilations in mSUGRA, JCAP 04 (2003) 001 [hep-ph/0301106] [INSPIRE].

[56] S. Raza, Q. Shafi and C.S. Ün, NLSP gluino and NLSP stop scenarios from b- $\tau$ Yukawa unification, Phys. Rev. D 92 (2015) 055010 [arXiv: 1412.7672] [INSPIRE].

[57] S. Mizuta and M. Yamaguchi, Coannihilation effects and relic abundance of Higgsino dominant LSPs, Phys. Lett. B 298 (1993) 120 [hep-ph/9208251] [INSPIRE].

[58] J. Edsjo and P. Gondolo, Neutralino relic density including coannihilations, Phys. Rev. D 56 (1997) 1879 [hep-ph/9704361] [INSPIRE].

[59] H. Baer, C. Balázs and A. Belyaev, Neutralino relic density in minimal supergravity with coannihilations, JHEP 03 (2002) 042 [hep-ph/0202076] [INSPIRE].

[60] A. Birkedal-Hansen and E.-h. Jeong, Gaugino and Higgsino coannihilations. 1. Neutralino neutralino interactions, JHEP 02 (2003) 047 [hep-ph/0210041] [INSPIRE].

[61] S. Profumo and C.E. Yaguna, Gluino coannihilations and heavy bino dark matter, Phys. Rev. D 69 (2004) 115009 [hep-ph/0402208] [INSPIRE].

[62] D. Feldman, Z. Liu and P. Nath, Gluino NLSP, Dark Matter via Gluino Coannihilation and LHC Signatures, Phys. Rev. D 80 (2009) 015007 [arXiv:0905.1148] [INSPIRE].

[63] N. Chen, D. Feldman, Z. Liu, P. Nath and G. Peim, Low Mass Gluino within the Sparticle Landscape, Implications for Dark Matter and Early Discovery Prospects at LHC-7, Phys. Rev. D 83 (2011) 035005 [arXiv: 1011.1246] [INSPIRE].

[64] I. Gogoladze, R. Khalid and Q. Shafi, Yukawa Unification and Neutralino Dark Matter in $\mathrm{SU}(4)_{c} \times \mathrm{SU}(2)_{L} \times \mathrm{SU}(2)_{R}$, Phys. Rev. D 79 (2009) 115004 [arXiv:0903.5204] [INSPIRE].

[65] I. Gogoladze, R. Khalid and Q. Shafi, Coannihilation Scenarios and Particle Spectroscopy in $\mathrm{SU}(4)_{c} \times \mathrm{SU}(2)_{L} \times \mathrm{SU}(2)_{R}$, Phys. Rev. D 80 (2009) 095016 [arXiv: 0908.0731] [inSPIRE].

[66] M. Adeel Ajaib, T. Li, Q. Shafi and K. Wang, NLSP Gluino Search at the Tevatron and early LHC, JHEP 01 (2011) 028 [arXiv: 1011.5518] [INSPIRE].

[67] K. Harigaya, M. Ibe and T.T. Yanagida, A Closer Look at Gaugino Masses in Pure Gravity Mediation Model/Minimal Split SUSY Model, JHEP 12 (2013) 016 [arXiv:1310.0643] [INSPIRE].

[68] K. Harigaya, K. Kaneta and S. Matsumoto, Gaugino coannihilations, Phys. Rev. D 89 (2014) 115021 [arXiv: 1403.0715] [INSPIRE].

[69] J.L. Evans and K.A. Olive, Universality in Pure Gravity Mediation with Vector Multiplets, Phys. Rev. D 90 (2014) 115020 [arXiv:1408.5102] [INSPIRE].

[70] A. De Simone, G.F. Giudice and A. Strumia, Benchmarks for Dark Matter Searches at the LHC, JHEP 06 (2014) 081 [arXiv: 1402.6287] [INSPIRE].

[71] M. Low and L.-T. Wang, Neutralino dark matter at 14 TeV and 100 TeV, JHEP 08 (2014) 161 [arXiv: 1404.0682] [INSPIRE].

[72] J.R. Ellis, F. Luo and K.A. Olive, Gluino Coannihilation Revisited, JHEP 09 (2015) 127 [arXiv: 1503.07142] [INSPIRE].

[73] G.L. Kane, C.F. Kolda, L. Roszkowski and J.D. Wells, Study of constrained minimal supersymmetry, Phys. Rev. D 49 (1994) 6173 [hep-ph/9312272] [INSPIRE].

[74] J.R. Ellis, T. Falk, K.A. Olive and M. Schmitt, Supersymmetric dark matter in the light of LEP-1.5, Phys. Lett. B 388 (1996) 97 [hep-ph/9607292] [INSPIRE]. 
[75] J.R. Ellis, T. Falk, K.A. Olive and M. Schmitt, Constraints on neutralino dark matter from LEP-2 and cosmology, Phys. Lett. B 413 (1997) 355 [hep-ph/9705444] [INSPIRE].

[76] V.D. Barger and C. Kao, Relic density of neutralino dark matter in supergravity models, Phys. Rev. D 57 (1998) 3131 [hep-ph/9704403] [INSPIRE].

[77] L. Roszkowski, R. Ruiz de Austri and T. Nihei, New cosmological and experimental constraints on the CMSSM, JHEP 08 (2001) 024 [hep-ph/0106334] [INSPIRE].

[78] A. Djouadi, M. Drees and J.L. Kneur, Constraints on the minimal supergravity model and prospects for SUSY particle production at future linear $e^{+} e^{-}$colliders, JHEP 08 (2001) 055 [hep-ph/0107316] [INSPIRE].

[79] U. Chattopadhyay, A. Corsetti and P. Nath, Supersymmetric dark matter and Yukawa unification, Phys. Rev. D 66 (2002) 035003 [hep-ph/0201001] [INSPIRE].

[80] J.R. Ellis, K.A. Olive and Y. Santoso, Constraining supersymmetry, New J. Phys. 4 (2002) 32 [hep-ph/0202110] [INSPIRE].

[81] H. Baer, C. Balázs, A. Belyaev, J.K. Mizukoshi, X. Tata and Y. Wang, Updated constraints on the minimal supergravity model, JHEP 07 (2002) 050 [hep-ph/0205325] [INSPIRE].

[82] R.L. Arnowitt and B. Dutta, Dark matter, muon $g-2$ and other accelerator constraints, hep-ph/0211417 [INSPIRE].

[83] J.R. Ellis, T. Falk, G. Ganis, K.A. Olive and M. Schmitt, Charginos and neutralinos in the light of radiative corrections: Sealing the fate of Higgsino dark matter, Phys. Rev. D 58 (1998) 095002 [hep-ph/9801445] [INSPIRE].

[84] J.R. Ellis, T. Falk, G. Ganis and K.A. Olive, Supersymmetric dark matter in the light of LEP and the Tevatron collider, Phys. Rev. D 62 (2000) 075010 [hep-ph/0004169] [INSPIRE].

[85] J.R. Ellis, K.A. Olive, Y. Santoso and V.C. Spanos, Supersymmetric dark matter in light of WMAP, Phys. Lett. B 565 (2003) 176 [hep-ph/0303043] [INSPIRE].

[86] H. Baer and C. Balázs, $\chi^{2}$ analysis of the minimal supergravity model including WMAP, $(g-2)_{\mu}$ and $b \rightarrow s \gamma$ constraints, JCAP 05 (2003) 006 [hep-ph/0303114] [INSPIRE].

[87] A.B. Lahanas and D.V. Nanopoulos, WMAPing out supersymmetric dark matter and phenomenology, Phys. Lett. B 568 (2003) 55 [hep-ph/0303130] [INSPIRE].

[88] U. Chattopadhyay, A. Corsetti and P. Nath, WMAP constraints, SUSY dark matter and implications for the direct detection of SUSY, Phys. Rev. D 68 (2003) 035005 [hep-ph/0303201] [INSPIRE].

[89] C. Muñoz, Dark matter detection in the light of recent experimental results, Int. J. Mod. Phys. A 19 (2004) 3093 [hep-ph/0309346] [INSPIRE].

[90] R.L. Arnowitt, B. Dutta and B. Hu, Dark matter, muon $g-2$ and other SUSY constraints, hep-ph/0310103 [INSPIRE].

[91] J.R. Ellis and K.A. Olive, Supersymmetric Dark Matter Candidates, in Particle dark matter: observations, models and searches, G. Bertone ed., Cambridge Universiry Press (2010), pp. 142-163 [arXiv:1001.3651] [INSPIRE].

[92] O. Buchmueller et al., Implications of Improved Higgs Mass Calculations for Supersymmetric Models, Eur. Phys. J. C 74 (2014) 2809 [arXiv:1312.5233] [INSPIRE].

[93] D. Matalliotakis and H.P. Nilles, Implications of nonuniversality of soft terms in supersymmetric grand unified theories, Nucl. Phys. B 435 (1995) 115 [hep-ph/9407251] [INSPIRE]. 
[94] M. Olechowski and S. Pokorski, Electroweak symmetry breaking with nonuniversal scalar soft terms and large $\tan \beta$ solutions, Phys. Lett. B 344 (1995) 201 [hep-ph/9407404] [INSPIRE].

[95] V. Berezinsky, A. Bottino, J.R. Ellis, N. Fornengo, G. Mignola and S. Scopel, Neutralino dark matter in supersymmetric models with nonuniversal scalar mass terms, Astropart. Phys. 5 (1996) 1 [hep-ph/9508249] [INSPIRE].

[96] M. Drees, M.M. Nojiri, D.P. Roy and Y. Yamada, Light Higgsino dark matter, Phys. Rev. D 56 (1997) 276 [Erratum ibid. D 64 (2001) 039901] [hep-ph/9701219] [INSPIRE].

[97] M. Drees, Y.G. Kim, M.M. Nojiri, D. Toya, K. Hasuko and T. Kobayashi, Scrutinizing LSP dark matter at the CERN LHC, Phys. Rev. D 63 (2001) 035008 [hep-ph/0007202] [INSPIRE].

[98] P. Nath and R.L. Arnowitt, Nonuniversal soft SUSY breaking and dark matter, Phys. Rev. D 56 (1997) 2820 [hep-ph/9701301] [INSPIRE].

[99] A. Bottino, F. Donato, N. Fornengo and S. Scopel, Probing the supersymmetric parameter space by WIMP direct detection, Phys. Rev. D 63 (2001) 125003 [hep-ph/0010203] [INSPIRE].

[100] S. Profumo, Neutralino dark matter, $b-\tau$ Yukawa unification and nonuniversal sfermion masses, Phys. Rev. D 68 (2003) 015006 [hep-ph/0304071] [INSPIRE].

[101] D.G. Cerdeno and C. Muñoz, Neutralino dark matter in supergravity theories with non-universal scalar and gaugino masses, JHEP 10 (2004) 015 [hep-ph/0405057] [INSPIRE].

[102] J.R. Ellis, K.A. Olive and Y. Santoso, The MSSM parameter space with nonuniversal Higgs masses, Phys. Lett. B 539 (2002) 107 [hep-ph/0204192] [InSPIRE].

[103] J.R. Ellis, T. Falk, K.A. Olive and Y. Santoso, Exploration of the MSSM with nonuniversal Higgs masses, Nucl. Phys. B 652 (2003) 259 [hep-ph/0210205] [INSPIRE].

[104] H. Baer, A. Mustafayev, S. Profumo, A. Belyaev and X. Tata, Neutralino cold dark matter in a one parameter extension of the minimal supergravity model, Phys. Rev. D 71 (2005) 095008 [hep-ph/0412059] [INSPIRE].

[105] H. Baer, A. Mustafayev, S. Profumo, A. Belyaev and X. Tata, Direct, indirect and collider detection of neutralino dark matter in SUSY models with non-universal Higgs masses, JHEP 07 (2005) 065 [hep-ph/0504001] [INSPIRE].

[106] J.R. Ellis, K.A. Olive and P. Sandick, Varying the Universality of Supersymmetry-Breaking Contributions to MSSM Higgs Boson Masses, Phys. Rev. D 78 (2008) 075012 [arXiv:0805.2343] [INSPIRE].

[107] G.F. Giudice and A. Strumia, Probing High-Scale and Split Supersymmetry with Higgs Mass Measurements, Nucl. Phys. B 858 (2012) 63 [arXiv:1108.6077] [InSPIRE].

[108] E. Bagnaschi, G.F. Giudice, P. Slavich and A. Strumia, Higgs Mass and Unnatural Supersymmetry, JHEP 09 (2014) 092 [arXiv: 1407.4081] [INSPIRE].

[109] G. Degrassi, S. Heinemeyer, W. Hollik, P. Slavich and G. Weiglein, Towards high precision predictions for the MSSM Higgs sector, Eur. Phys. J. C 28 (2003) 133 [hep-ph/0212020] [INSPIRE].

[110] S. Heinemeyer, W. Hollik and G. Weiglein, The Masses of the neutral $\mathcal{C} \mathcal{P}$-even Higgs bosons in the MSSM: Accurate analysis at the two loop level, Eur. Phys. J. C 9 (1999) 343 [hep-ph/9812472] [INSPIRE].

[111] S. Heinemeyer, W. Hollik and G. Weiglein, FeynHiggs: A Program for the calculation of the masses of the neutral CP even Higgs bosons in the MSSM, Comput. Phys. Commun. 124 (2000) 76 [hep-ph/9812320] [INSPIRE]. 
[112] M. Frank, T. Hahn, S. Heinemeyer, W. Hollik, H. Rzehak and G. Weiglein, The Higgs Boson Masses and Mixings of the Complex MSSM in the Feynman-Diagrammatic Approach, JHEP 02 (2007) 047 [hep-ph/0611326] [INSPIRE].

[113] T. Hahn, S. Heinemeyer, W. Hollik, H. Rzehak and G. Weiglein, High-Precision Predictions for the Light CP-Even Higgs Boson Mass of the Minimal Supersymmetric Standard Model, Phys. Rev. Lett. 112 (2014) 141801 [arXiv:1312.4937] [INSPIRE].

[114] FeynHiggs: overview, http://www.feynhiggs.de.

[115] E. Dudas, A. Linde, Y. Mambrini, A. Mustafayev and K.A. Olive, Strong moduli stabilization and phenomenology, Eur. Phys. J. C 73 (2013) 2268 [arXiv:1209.0499] [INSPIRE].

[116] J.L. Feng, K.T. Matchev and T. Moroi, Multi-TeV scalars are natural in minimal supergravity, Phys. Rev. Lett. 84 (2000) 2322 [hep-ph/9908309] [INSPIRE].

[117] J.L. Feng, K.T. Matchev and T. Moroi, Focus points and naturalness in supersymmetry, Phys. Rev. D 61 (2000) 075005 [hep-ph/9909334] [INSPIRE].

[118] J.L. Feng, K.T. Matchev and F. Wilczek, Neutralino dark matter in focus point supersymmetry, Phys. Lett. B 482 (2000) 388 [hep-ph/0004043] [inSPIRE].

[119] H. Baer, T. Krupovnickas, S. Profumo and P. Ullio, Model independent approach to focus point supersymmetry: From dark matter to collider searches, JHEP 10 (2005) 020 [hep-ph/0507282] [INSPIRE].

[120] J.L. Feng, K.T. Matchev and D. Sanford, Focus Point Supersymmetry Redux, Phys. Rev. D 85 (2012) 075007 [arXiv: 1112.3021] [INSPIRE].

[121] P. Draper, J.L. Feng, P. Kant, S. Profumo and D. Sanford, Dark Matter Detection in Focus Point Supersymmetry, Phys. Rev. D 88 (2013) 015025 [arXiv: 1304.1159] [InSPIRE].

[122] N. Arkani-Hamed, A. Delgado and G.F. Giudice, The Well-tempered neutralino, Nucl. Phys. B 741 (2006) 108 [hep-ph/0601041] [INSPIRE].

[123] H. Baer, A. Mustafayev, E.-K. Park, S. Profumo and X. Tata, Mixed Higgsino dark matter from a reduced $\mathrm{SU}(3)$ gaugino mass: Consequences for dark matter and collider searches, JHEP 04 (2006) 041 [hep-ph/0603197] [INSPIRE].

[124] M. Ibe, T. Moroi and T.T. Yanagida, Possible Signals of Wino LSP at the Large Hadron Collider, Phys. Lett. B 644 (2007) 355 [hep-ph/0610277] [INSPIRE].

[125] M. Ibe and T.T. Yanagida, The Lightest Higgs Boson Mass in Pure Gravity Mediation Model, Phys. Lett. B 709 (2012) 374 [arXiv:1112.2462] [inSPIRE].

[126] M. Ibe, S. Matsumoto and T.T. Yanagida, Pure Gravity Mediation with $m_{3 / 2}=10-100 \mathrm{TeV}$, Phys. Rev. D 85 (2012) 095011 [arXiv: 1202.2253] [INSPIRE].

[127] L.J. Hall and Y. Nomura, Spread Supersymmetry, JHEP 01 (2012) 082 [arXiv:1111.4519] [INSPIRE].

[128] N. Arkani-Hamed, A. Gupta, D.E. Kaplan, N. Weiner and T. Zorawski, Simply Unnatural Supersymmetry, arXiv:1212.6971 [INSPIRE].

[129] A. Arvanitaki, N. Craig, S. Dimopoulos and G. Villadoro, Mini-Split, JHEP 02 (2013) 126 [arXiv: 1210.0555] [INSPIRE].

[130] L.J. Hall, Y. Nomura and S. Shirai, Spread Supersymmetry with Wino LSP: Gluino and Dark Matter Signals, JHEP 01 (2013) 036 [arXiv:1210.2395] [INSPIRE].

[131] J.L. Evans, M. Ibe, K.A. Olive and T.T. Yanagida, Universality in Pure Gravity Mediation, Eur. Phys. J. C 73 (2013) 2468 [arXiv:1302.5346] [INSPIRE]. 
[132] G.F. Giudice and A. Masiero, A Natural Solution to the $\mu$-Problem in Supergravity Theories, Phys. Lett. B 206 (1988) 480 [INSPIRE].

[133] K. Inoue, M. Kawasaki, M. Yamaguchi and T.T. Yanagida, Vanishing squark and slepton masses in a class of supergravity models, Phys. Rev. D 45 (1992) 328 [INSPIRE].

[134] E. Dudas, Y. Mambrini, A. Mustafayev and K.A. Olive, Relating the CMSSM and SUGRA Models with GUT Scale and Super-GUT Scale Supersymmetry Breaking, Eur. Phys. J. C 72 (2012) 2138 [Erratum ibid. C 73 (2013) 2430] [arXiv: 1205.5988] [INSPIRE].

[135] R. Barbieri, S. Ferrara and C.A. Savoy, Gauge Models with Spontaneously Broken Local Supersymmetry, Phys. Lett. B 119 (1982) 343 [INSPIRE].

[136] V.D. Barger, M.S. Berger and P. Ohmann, The Supersymmetric particle spectrum, Phys. Rev. D 49 (1994) 4908 [hep-ph/9311269] [InSPIRE].

[137] W. de Boer, R. Ehret and D.I. Kazakov, Predictions of SUSY masses in the minimal supersymmetric grand unified theory, Z. Phys. C 67 (1995) 647 [hep-ph/9405342] [INSPIRE].

[138] M. Carena, J.R. Ellis, A. Pilaftsis and C.E.M. Wagner, Higgs boson pole masses in the MSSM with explicit CP-violation, Nucl. Phys. B 625 (2002) 345 [hep-ph/0111245] [INSPIRE].

[139] A.E. Nelson and N. Seiberg, $R$ symmetry breaking versus supersymmetry breaking, Nucl. Phys. B 416 (1994) 46 [hep-ph/9309299] [INSPIRE].

[140] M. Dine and D. MacIntire, Supersymmetry, naturalness and dynamical supersymmetry breaking, Phys. Rev. D 46 (1992) 2594 [hep-ph/9205227] [INSPIRE].

[141] L. Randall and R. Sundrum, Out of this world supersymmetry breaking, Nucl. Phys. B 557 (1999) 79 [hep-th/9810155] [INSPIRE].

[142] G.F. Giudice, M.A. Luty, H. Murayama and R. Rattazzi, Gaugino mass without singlets, JHEP 12 (1998) 027 [hep-ph/9810442] [INSPIRE].

[143] J.A. Bagger, T. Moroi and E. Poppitz, Anomaly mediation in supergravity theories, JHEP 04 (2000) 009 [hep-th/9911029] [INSPIRE].

[144] P. Binetruy, M.K. Gaillard and B.D. Nelson, One loop soft supersymmetry breaking terms in superstring effective theories, Nucl. Phys. B 604 (2001) 32 [hep-ph/0011081] [INSPIRE].

[145] J.D. Wells, Implications of supersymmetry breaking with a little hierarchy between gauginos and scalars, hep-ph/0306127 [INSPIRE].

[146] N. Arkani-Hamed and S. Dimopoulos, Supersymmetric unification without low energy supersymmetry and signatures for fine-tuning at the LHC, JHEP 06 (2005) 073 [hep-th/0405159] [INSPIRE].

[147] G.F. Giudice and A. Romanino, Split supersymmetry, Nucl. Phys. B 699 (2004) 65 [Erratum ibid. B 706 (2005) 65] [hep-ph/0406088] [INSPIRE].

[148] N. Arkani-Hamed, S. Dimopoulos, G.F. Giudice and A. Romanino, Aspects of split supersymmetry, Nucl. Phys. B 709 (2005) 3 [hep-ph/0409232] [INSPIRE].

[149] J.D. Wells, PeV-scale supersymmetry, Phys. Rev. D 71 (2005) 015013 [hep-ph/0411041] [INSPIRE].

[150] N. Nagata, H. Otono and S. Shirai, Probing bino-gluino coannihilation at the LHC, Phys. Lett. B 748 (2015) 24 [arXiv: 1504.00504] [INSPIRE]. 